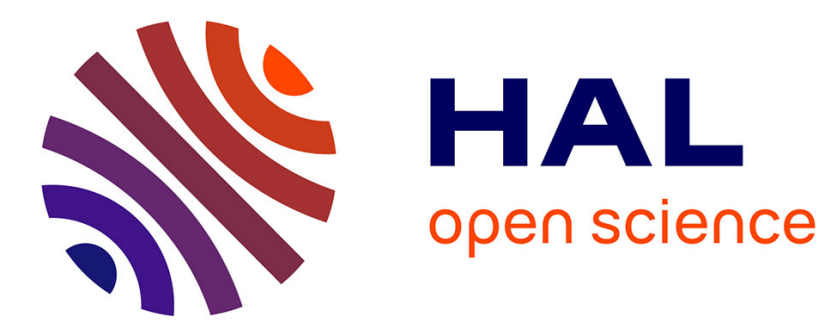

\title{
Uncertainty analysis in fault tree models with dependent basic events
}

Nicola Pedroni, Enrico Zio

\section{To cite this version:}

Nicola Pedroni, Enrico Zio. Uncertainty analysis in fault tree models with dependent basic events. Risk Analysis, 2013, 33 (6), pp.1146-1173. hal-00839643

\section{HAL Id: hal-00839643 \\ https://hal-centralesupelec.archives-ouvertes.fr/hal-00839643}

Submitted on 28 Jun 2013

HAL is a multi-disciplinary open access archive for the deposit and dissemination of scientific research documents, whether they are published or not. The documents may come from teaching and research institutions in France or abroad, or from public or private research centers.
L'archive ouverte pluridisciplinaire HAL, est destinée au dépôt et à la diffusion de documents scientifiques de niveau recherche, publiés ou non, émanant des établissements d'enseignement et de recherche français ou étrangers, des laboratoires publics ou privés. 


\title{
Uncertainty analysis in fault tree models with dependent
}

\section{basic events}

\author{
Nicola Pedroni ${ }^{1}$, Enrico Zio ${ }^{2,3, *}$ \\ ${ }^{1}$ Energy Department, Politecnico di Milano \\ Via Ponzio, 34/3 - 20133 Milano, Italy \\ Fax: +39-02-2399-6309; Phone: +39-02-2399-6372 \\ E-mail address: nicola.pedroni@mail.polimi.it \\ ${ }^{2}$ Energy Department, Politecnico di Milano \\ Via Ponzio, 34/3 - 20133 Milano, Italy \\ Fax: +39-02-2399-6309; Phone: +39-02-2399-6340 \\ E-mail address: enrico.zio@polimi.it
}

${ }^{3}$ Chair of system science and the energetic challenge, Electricitè de France-Ecole Centrale de Paris and Supelec

Grande Voie des Vignes, 92295, Chatenay Malabry-Cedex, France

Fax: +33-01-41-13-12-72; Phone: +33-01-41-13-16-06

E-mail address 1: enrico.zio@ecp.fr

E-mai address 2: enrico.zio@supelec.fr

*Corresponding author 


\begin{abstract}
In general, two types of dependence need to be considered when estimating the probability of the Top Event (TE) of a Fault Tree (FT): 'objective' dependence between the (random) occurrences of different Basic Events (BEs) in the FT and 'state-of-knowledge' (epistemic) dependence between estimates of the epistemically-uncertain probabilities of some BEs of the FT model.

In this paper, we study the effects on the TE probability of objective and epistemic dependences. The well-known Frèchet bounds and the Distribution Envelope Determination (DEnv) method are used to model all kinds of (possibly unknown) objective and epistemic dependences, respectively.

For exemplification, the analyses are carried out on a FT with six BEs. Results show that both types of dependence significantly affect the TE probability; however, the effects of epistemic dependence are likely to be overwhelmed by those of objective dependence (if present).
\end{abstract}

KEYWORDS: fault tree; epistemically-uncertain probabilities; objective and epistemic dependences. 


\section{INTRODUCTION}

In Fault Tree Analysis (FTA) ${ }^{(1-5)}$, limiting relative frequency probabilities are typically used to describe aleatory uncertainty and subjective probabilities to describe epistemic uncertainty ${ }^{1}$ (2, 6-14). Recently, it has been argued that a probabilistic representation of epistemic uncertainty is difficult to justify in those cases in which the analysis is carried out based on insufficient knowledge, information and data. To overcome this hurdle, a number of alternative nonprobabilistic representation frameworks have been proposed ${ }^{(15-19)}$, e.g., fuzzy set theory ${ }^{(20-28)}$, possibility theory ${ }^{(29-33)}$, hybrid combinations of probability and possibility theories ${ }^{(30,34-36)}$, Dempster-Shafer (DS) theory of evidence ${ }^{(37-44)}$ and interval analysis ${ }^{(45-49)}$.

To describe the epistemic uncertainty in the probabilities (chances) of the Basic Events (BEs) of a Fault Tree (FT) model, here we use possibility distributions and DS structures, together with probability distributions. The epistemic uncertainties are then propagated onto the probability (chance) of the Top Event (TE) by resorting to the general and comprehensive framework of DS theory of evidence ${ }^{(37-44)}$.

Dependence may exist among some BEs of the FT model ${ }^{(40)}$. In particular, two types of dependence need to be considered. The first type relates to the (dependent) occurrence of different (random) BEs (in the following, this kind of dependence will be referred to as 'objective' or 'aleatory'). An example of this objective (aleatory) dependence may be represented by the occurrence of multiple failures which result directly from a common or shared root cause (e.g., extreme environmental conditions, failure of a piece of hardware external to the system, or a human error): they are termed Common Cause Failures (CCFs)

\footnotetext{
${ }^{1}$ In the following, 'probability' refers to the limiting relative frequency concept whenever followed by the word 'chance' in parenthesis, and to the epistemic concept whenever used alone.
} 
and frequently affect, e.g., identical components in redundant trains of a safety system ${ }^{(2,50-52)}$; another example is that of cascading failures, i.e., multiple failures initiated by the failure of one component in the system, as a sort of chain reaction or domino effect ${ }^{(52-57)}$. The second type refers to the dependence possibly existing between the estimates of the epistemicallyuncertain probabilities (chances) of some BEs of the FT model (in the following, this kind of dependence will be referred to as 'state-of-knowledge' or 'epistemic'). This state-ofknowledge (epistemic) dependence exists when the probabilities (chances) of some BEs are estimated by resorting to dependent information sources (e.g., to the same experts/observers or to correlated data sets $)^{(2,11)}$.

In this context, the aim of the present paper is to systematically analyze and quantify the effects of objective (aleatory) and state-of-knowledge (epistemic) dependences between the BEs on the TE probability (chance). In more details, the following analyses are performed:

1. the study of the effects of different states of objective dependence between the BEs when the state of epistemic dependence between the BE probabilities (chances) is defined. In this analysis the well-known Frèchet bounds ${ }^{(40,58-60)}$ are used to model the full range of objective dependences here of interest;

2. the study of the effects of different states of epistemic dependence between the BE probabilities (chances) when the state of objective dependence between the BEs is given. In this analysis the Distribution Envelope Determination (DEnv) method ${ }^{(61-65)}$ is undertaken in order to account for all kinds of (possibly unknown) epistemic dependences between the BE probabilities (chances).

To keep the analysis simple and thus retain a clear view of each step, the investigations are carried out with respect to an example involving a FT with six BEs; different numerical indicators are considered to perform a fair and quantitative comparison between different 
states of objective and epistemic dependence and evaluate their effects on the TE probability (chance).

The work benefits from the efforts that have already been done to address objective and stateof-knowledge dependences in FTA. In ${ }^{(66-68)}$ objective dependencies between BEs are treated by means of alpha factor models within the traditional framework of Common Cause Failure $(\mathrm{CCF})$ analysis. In ${ }^{(40)}$ and ${ }^{(60)}$ the use of Frank copula and Pearson correlation coefficient is proposed to describe a wide range of objective dependences between the BEs. In ${ }^{(69)}$ and ${ }^{(70)}$ (fuzzy) dependency factors are employed to model dependent BEs. In ${ }^{(71-74)}$ state-ofknowledge dependences between the BE probabilities (chances) are described by traditional correlation coefficients and propagated by the method of moments. In ${ }^{(68)}$ and ${ }^{(75)}$ statistical epistemic correlations are modeled by resorting to the Nataf transformation ${ }^{(76)}$ within a traditional Monte Carlo Simulation (MCS) framework ${ }^{(77,78)}$. Finally, in ${ }^{(79)}$ the Dependency Bound Convolution (DBC) approach is undertaken to account for all kinds of (possibly unknown) epistemic dependences between the probabilities (chances) of correlated BEs.

The remainder of the paper is organized as follows. In Section 2, the methods employed in this study to model objective and state-of-knowledge dependences in FTA are described; in Section 3, the FT studied is presented; in Section 4, the results of the application of the methods of Section 2 to the FT of Section 3 are shown; finally, Section 5 offers some discussions and conclusions. 


\section{METHODS EMPLOYED IN THIS STUDY FOR MODELING DEPENDENCES IN FAULT TREE ANALYSIS}

In this Section, the computational strategies here employed for modeling dependences in Fault Tree Analysis (FTA) are described in detail: in particular, Section 2.1 deals with the representation of objective (aleatory) dependences between (the occurrence of) Basic Events (BEs); instead, Section 2.2 concerns the treatment of state-of-knowledge (epistemic) dependences between the probabilities (chances) of the BEs.

Other approaches for modeling objective dependences between (random) events can be found in ${ }^{(40,60,66-70)}$.

\subsection{Modeling objective (aleatory) dependences between the basic events}

Let $B_{1}$ and $B_{2}$ be two BEs with probabilities (chances) $P\left(B_{1}\right)$ and $P\left(B_{2}\right)$, respectively; with reference to the simple parallel and series systems of Figure 1 (left and right, respectively), $B_{1}$ and $B_{2}$ may represent the events of failure of Components 1 and 2, respectively, and $P\left(B_{1}\right)$ and $P\left(B_{2}\right)$ the corresponding probabilities (chances). If $B_{1}$ and $B_{2}$ are independent, the occurrence of one event (e.g., failure of Component 1) does not affect the occurrence of the other (e.g., failure of Component 2), i.e., $P\left(B_{1} \mid B_{2}\right)=P\left(B_{1}\right)$ and $P\left(B_{2} \mid B_{1}\right)=P\left(B_{2}\right)$. Then, the probabilities (chances) $P\left(B_{1} \cap_{\text {ind }} B_{2}\right)$ and $P\left(B_{1} \cup_{\text {ind }} B_{2}\right)$ of the conjunction $\left(B_{1} \cap_{\text {ind }} B_{2}\right)$ and disjunction $\left(B_{1} \cup_{\text {ind }} B_{2}\right.$ ) of events $B_{1}$ and $B_{2}$ (i.e., the probabilities-chances of failure of the parallel and series systems of Figure 1, left and right, respectively) are given by the wellknown deterministic functions $g_{B_{1} \cap_{\text {ind }} B_{2}}\left(P\left(B_{1}\right), P\left(B_{2}\right)\right)(1)$ and $g_{B_{1} \cup_{\text {ind }} B_{2}}\left(P\left(B_{1}\right), P\left(B_{2}\right)\right)$ respectively ${ }^{(40,60)}$ :

$$
\begin{aligned}
& P\left(B_{1} \cap_{\text {ind }} B_{2}\right)=g_{B_{1} \cap_{\text {ind }} B_{2}}\left(P\left(B_{1}\right), P\left(B_{2}\right)\right)=P\left(B_{1}\right) \cdot P\left(B_{2}\right) \\
& P\left(B_{1} \cup_{\text {ind }} B_{2}\right)=g_{B_{1} \cup_{\text {ind }} B_{2}}\left(P\left(B_{1}\right), P\left(B_{2}\right)\right)=1-\left(1-P\left(B_{1}\right)\right) \cdot\left(1-P\left(B_{2}\right)\right)
\end{aligned}
$$


where the symbols ' $\cap_{\text {ind }}$ ' and ' $\cup_{\text {ind }}$ ' denote the conjunction and disjunction of independent events, respectively.

If events $B_{1}$ and $B_{2}$ are perfectly dependent (i.e., $B_{1} \subset B_{2}$ or $B_{2} \subset B_{1}$ ), the occurrence of one event (e.g., failure of Component 1 in Figure 1) implies the occurrence of the other (e.g., failure of Component 2 in Figure 1) (i.e., $P\left(B_{2} \mid B_{1}\right)=1$ or $P\left(B_{1} \mid B_{2}\right)=1$, respectively). In this

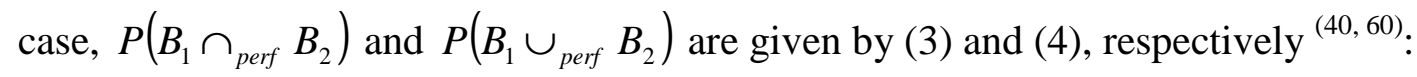

$$
\begin{aligned}
& P\left(B_{1} \cap_{\text {perf }} B_{2}\right)=g_{B_{1} \cap_{\text {perf }} B_{2}}\left(P\left(B_{1}\right), P\left(B_{2}\right)\right)=\min \left(P\left(B_{1}\right), P\left(B_{2}\right)\right) \\
& P\left(B_{1} \cup_{\text {perf }} B_{2}\right)=g_{B_{1} \cup_{\text {perf }} B_{2}}\left(P\left(B_{1}\right), P\left(B_{2}\right)\right)=\max \left(P\left(B_{1}\right), P\left(B_{2}\right)\right)
\end{aligned}
$$

where the symbols ' $\cap_{\text {perf }}$ ' and ' $\cup_{\text {perf }}$ ' denote the conjunction and disjunction of perfectly dependent events, respectively. Examples of perfect dependence can be found in many engineered systems. For example, some components may be subject to the same maintenance strategy and suffer a common mistake in the procedure, or may experience the same history of environmental conditions leading to failure. Such shared life conditions may make failures of components close to be perfectly dependent events $(2,40,50,51)$. The importance of this state of dependence can be understood with reference to the simple parallel system of Figure 1, left: if Components 1 and 2 were perfectly dependent, the failure of only one component would lead to the failure of the entire parallel system.

Finally, if events $B_{1}$ and $B_{2}$ are oppositely dependent, the occurrence of one event minimizes the likelihood of occurrence of the other. In this case, $P\left(B_{1} \cap_{\text {opp }} B_{2}\right)$ and $P\left(B_{1} \cup_{\text {opp }} B_{2}\right)$ are given by (5) and (6), respectively ${ }^{(40,60)}$ :

$$
\begin{aligned}
& P\left(B_{1} \cap_{\text {opp }} B_{2}\right)=g_{B_{1} \cap_{\text {opp }} B_{2}}\left(P\left(B_{1}\right), P\left(B_{2}\right)\right)=\max \left(P\left(B_{1}\right)+P\left(B_{2}\right)-1,0\right) \\
& P\left(B_{1} \cup_{\text {opp }} B_{2}\right)=g_{B_{1} \cup_{\text {opp }} B_{2}}\left(P\left(B_{1}\right), P\left(B_{2}\right)\right)=\min \left(P\left(B_{1}\right)+P\left(B_{2}\right), 1\right)
\end{aligned}
$$


where the symbols ' $\cap_{o p p}$ ' and ' $\cup_{o p p}$ ' denote the conjunction and disjunction of oppositely dependent events, respectively. An example of opposite dependence may be represented by the series of a fuse wire (e.g., Component 1 in Figure 1, right) and an electronic device (e.g., Component 2 in Figure 1, right). In case of overcurrent, failure of the fuse wire (event $B_{1}$ ) prevents failure of the electronic component (event $B_{2}$ ); thus, the joint failure of both components might be better modeled by events that are oppositely dependent than independent.

When no information at all about the state of objective dependence between events $B_{1}$ and $B_{2}$ is available, precise estimates for $P\left(B_{1} \cap B_{2}\right)$ and $P\left(B_{1} \cup B_{2}\right)$ cannot be computed. Instead, extreme bounds $P\left(B_{1} \cap_{u k n} B_{2}\right)$ (7) and $P\left(B_{1} \cup_{u k n} B_{2}\right)$ (8) on $P\left(B_{1} \cap B_{2}\right)$ and $P\left(B_{1} \cup B_{2}\right)$, respectively, can be obtained by means of the classical Frèchet inequalities ${ }^{(40,58-60)}$ :

$$
\begin{aligned}
& P\left(B_{1} \cap_{u k n} B_{2}\right)=\left[g_{B_{1} \cap_{\text {opp }} B_{2}}, g_{B_{1} \cap_{\text {perf }} B_{2}}\right]=\left[\max \left(P\left(B_{1}\right)+P\left(B_{2}\right)-1,0\right), \min \left(P\left(B_{1}\right), P\left(B_{2}\right)\right)\right] \\
& P\left(B_{1} \cup_{u k n} B_{2}\right)=\left[g_{B_{1} \cup_{\text {perf }} B_{2}}, g_{B_{1} \cup_{\text {opp }} B_{2}}\right]=\left[\max \left(P\left(B_{1}\right), P\left(B_{2}\right)\right), \min \left(P\left(B_{1}\right)+P\left(B_{2}\right), 1\right)\right]
\end{aligned}
$$

where functions $g_{B_{1} \cap_{\text {perf }} B_{2}}, g_{B_{1} \cup \text { perf } B_{2}}, g_{B_{1} \cap_{\text {opp }} B_{2}}$ and $g_{B_{1} \cup \text { opp }} B_{2}$ are defined in (3)-(6) and the symbols ' $\cap_{u k n}$ ' and ' $\cup_{u k n}$ ' denote the conjunction and disjunction of events whose state of objective dependence is completely unknown, respectively. As stated in ${ }^{(40)}$, it is worth mentioning that i) $P\left(B_{1} \cap_{u k n} B_{2}\right)$ (7) and $P\left(B_{1} \cup_{u k n} B_{2}\right)$ (8) are "bounds on all possible cases of objective dependence" (because they include by construction dependences ranging from opposite to perfect) and ii) they represent the "best possible bounds in the absence of information about objective dependence, i.e., they could not be any tighter without excluding some possible objective dependences" ${ }^{(40)}$. 
Finally, if the analyst is able to say something about the sign of objective dependence, then Frèchet bounds (7) and (8) can be tightened. In particular, if $B_{1}$ and $B_{2}$ are positively dependent, i.e., the occurrence of one event favors the occurrence of the other, then $P\left(B_{1} \mid B_{2}\right)>P\left(B_{1}\right) \quad$ and $\quad P\left(B_{2} \mid B_{1}\right)>P\left(B_{2}\right), \quad$ from which it follows that $P\left(B_{1} \cap_{p o s} B_{2}\right)>P\left(B_{1} \cap_{\text {ind }} B_{2}\right)$. In this case, bounds $P\left(B_{1} \cap_{p o s} B_{2}\right)$ and $P\left(B_{1} \cup_{\text {pos }} B_{2}\right)$ on $P\left(B_{1} \cap B_{2}\right)$ and $P\left(B_{1} \cup B_{2}\right)$ are obtained by (9) and (10), respectively ${ }^{(40,60)}$ :

$$
\begin{aligned}
& P\left(B_{1} \cap_{\text {pos }} B_{2}\right)=\left[g_{B_{1} \cap_{\text {ind }} B_{2}}, g_{B_{1} \cap_{\text {perf }} B_{2}}\right]=\left[P\left(B_{1}\right) \cdot P\left(B_{2}\right), \min \left(P\left(B_{1}\right), P\left(B_{2}\right)\right)\right] \\
& P\left(B_{1} \cup_{\text {pos }} B_{2}\right)=\left[g_{B_{1} \cup_{\text {perf }} B_{2}}, g_{B_{1} \cup_{\text {ind }} B_{2}}\right]=\left[\max \left(P\left(B_{1}\right), P\left(B_{2}\right)\right), 1-\left(1-P\left(B_{1}\right)\right) \cdot\left(1-P\left(B_{2}\right)\right)\right] .
\end{aligned}
$$

On the contrary, if $B_{1}$ and $B_{2}$ are negatively dependent, then bounds $P\left(B_{1} \cap_{\text {neg }} B_{2}\right)$ and $P\left(B_{1} \cup_{\text {neg }} B_{2}\right)$ on $P\left(B_{1} \cap B_{2}\right)$ and $P\left(B_{1} \cup B_{2}\right)$ are obtained using (11) and (12), respectively $(40,60)$.

$$
\begin{aligned}
& P\left(B_{1} \cap_{\text {neg }} B_{2}\right)=\left[g_{B_{1} \cap_{\text {opp }} B_{2}}, g_{B_{1} \cap_{\text {ind }} B_{2}}\right]=\left[\max \left(P\left(B_{1}\right)+P\left(B_{2}\right)-1,0\right), P\left(B_{1}\right) \cdot P\left(B_{2}\right)\right] \\
& P\left(B_{1} \cup_{\text {neg }} B_{2}\right)=\left[g_{B_{1} \cup_{\text {ind }} B_{2}}, g_{B_{1} \cup_{\text {opp }} B_{2}}\right]=\left[1-\left(1-P\left(B_{1}\right)\right) \cdot\left(1-P\left(B_{2}\right)\right), \min \left(P\left(B_{1}\right)+P\left(B_{2}\right), 1\right)\right] .
\end{aligned}
$$

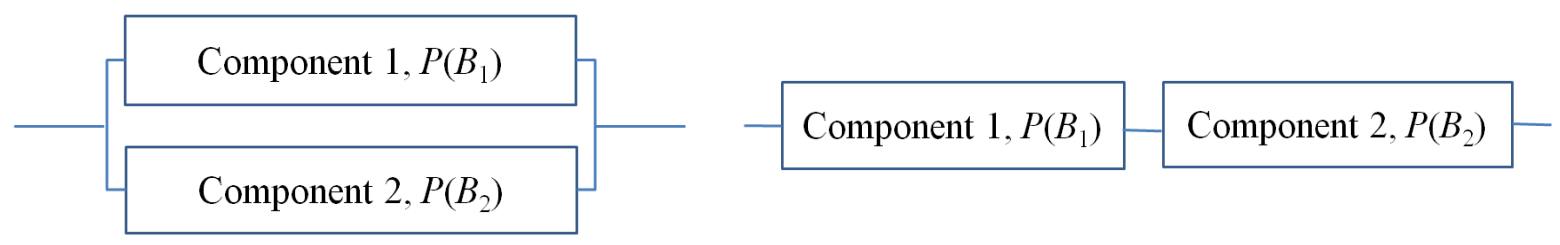

Figure 1. Simple parallel (left) and series (right) systems of two components whose failure probabilities (chances) are $P\left(B_{1}\right)$ and $P\left(B_{2}\right)$, respectively

\subsection{Modeling state-of-knowledge (epistemic) dependences between the probabilities (chances) of the basic events}

In all generality, let us assume that:

i. events $B_{1}$ and $B_{2}$ are linked to an event $Z$ of interest by the generic logical connection '○’ (e.g., ' $\circ$ ' may stand for ' $\cap$ ', ‘ $\cup$ ', ...); 
ii. the state of objective dependence between events $B_{1}$ and $B_{2}$ is defined and indicated as ' $\bigcirc_{o b j}$ ' for example, if there is positive objective dependence between $B_{1}$ and $B_{2}$, then the subscript 'obj' stands for 'pos' (see the previous Section 2.1);

iii. the probability (chance) $P(Z)$ of the event $Z=\left(B_{1} \circ_{o b j} B_{2}\right)$ of interest is obtained as $P(Z)=g_{Z}\left(P\left(B_{1}\right), P\left(B_{2}\right)\right)$, where $g_{Z}\left(P\left(B_{1}\right), P\left(B_{2}\right)\right)$ is a deterministic function which provides a formal, mathematical description of the state of objective dependence between events $B_{1}$ and $B_{2}$ (for example, $g_{Z}(\cdot, \cdot)$ may be one of those reported in (1)(12))

iv. the probabilities (chances) $P\left(B_{1}\right)$ and $P\left(B_{2}\right)$ of events $B_{1}$ and $B_{2}$ are considered epistemically-uncertain. For ease of explanation, let us suppose that the epistemic uncertainty on $P\left(B_{1}\right)$ and $P\left(B_{2}\right)$ is represented by the Dempster-Shafer (DS) structures $\quad\left\{\left(A_{P\left(B_{1}\right)}^{i}, m\left(A_{P\left(B_{1}\right)}^{i}\right)\right): i=1,2, \ldots, n_{B_{1}}\right\} \quad$ and $\quad\left\{\left(A_{P\left(B_{2}\right)}^{j}, m\left(A_{P\left(B_{2}\right)}^{j}\right)\right): j=1,2, \ldots, n_{B_{2}}\right\}$, respectively: in other words, $P\left(B_{1}\right)$ and $P\left(B_{2}\right)$ are described by two sets of $n_{B_{1}}$ and $n_{B_{2}}$ intervals (focal elements) $A_{P\left(B_{1}\right)}^{i}=\left[\underline{p}_{B_{1}}^{i}, \bar{p}_{B_{1}}^{i}\right], \quad i=1,2, \ldots, n_{B_{1}}$, and $A_{P\left(B_{2}\right)}^{j}=\left[\underline{p}_{B_{2}}^{j}, \bar{p}_{B_{2}}^{j}\right], j=1,2, \ldots, n_{B_{2}}$, respectively, each of which is assigned a probability (or belief) mass $m\left(A_{P\left(B_{1}\right)}^{i}\right), i=1,2, \ldots, n_{B_{1}}$, and $m\left(A_{P\left(B_{2}\right)}^{j}\right), j=1,2, \ldots, n_{B_{2}}$, respectively (it is worth stressing that $m\left(A_{P\left(B_{1}\right)}^{i}\right)$ and $m\left(A_{P\left(B_{2}\right)}^{j}\right)$ represent the degrees of belief of membership of $P\left(B_{1}\right)$ and $P\left(B_{2}\right)$ in sets $A_{P\left(B_{1}\right)}^{i}$ and $A_{P\left(B_{2}\right)}^{j}$ only, but without any specification of how these degrees of belief might be apportioned over $A_{P\left(B_{1}\right)}^{i}$ and $A_{P\left(B_{2}\right)}^{j}$, respectively; in other words, $m\left(A_{P\left(B_{1}\right)}^{i}\right)$ and $m\left(A_{P\left(B_{2}\right)}^{j}\right)$ express the proportion to which all available and relevant evidence supports the claim that $P\left(B_{1}\right)$ and $P\left(B_{2}\right)$, whose characterization is incomplete, belong to sets $A_{P\left(B_{1}\right)}^{i}$ and $A_{P\left(B_{2}\right)}^{j}$, respectively). 
By way of example, let $\left\{\left(A_{P\left(B_{1}\right)}^{i}, m\left(A_{P\left(B_{1}\right)}^{i}\right)\right): i=1,2, \ldots, n_{B_{1}}=2\right\} \quad=$ $\{([0.20,0.50], 0.35),([0.40,0.60], 0.65)\} \quad$ and $\quad\left\{\left(A_{P\left(B_{2}\right)}^{j}, m\left(A_{P\left(B_{2}\right)}^{j}\right)\right): j=1,2, \ldots, n_{B_{2}}=2\right\}=$ $\{([0.10,0.35], 0.45),([0.30,0.45], 0.55)\}$ : for clarity, the corresponding DS structures are pictorially shown in Figure 2, top left and right, respectively. Referring to probability (chance) $P\left(B_{1}\right)$ of event $B_{1}$ (Figure 2, top left), the corresponding DS structure can be interpreted as follows: probability (chance) $P\left(B_{1}\right)$ of event $B_{1}$ lies within interval $A_{P\left(B_{1}\right)}^{1}$ $=[0.20,0.50]$ at least with probability $m\left(A_{P\left(B_{1}\right)}^{1}\right)=0.35$, whereas it lies within interval $A_{P\left(B_{1}\right)}^{2}=[0.40,0.60]$ at least with probability $m\left(A_{P\left(B_{1}\right)}^{2}\right)=0.65$. Notice that the DS structures described above can be transformed into upper and lower Cumulative Distribution Functions (CDFs) $\bar{F}^{P\left(B_{1}\right)}, \bar{F}^{P\left(B_{2}\right)}, \underline{F}^{P\left(B_{1}\right)}$ and $\underline{F}^{P\left(B_{2}\right)}$ for $P\left(B_{1}\right)$ and $P\left(B_{2}\right)$, respectively: in particular, $\bar{F}^{P\left(B_{1}\right)}\left(p_{B_{1}}\right)=\bar{P}\left[P\left(B_{1}\right)<p_{B_{1}}\right]=\sum_{A_{P\left(B_{1}\right)}^{i} \cap\left\{0, p_{B_{1}} \neq 0\right.} m\left(A_{P\left(B_{1}\right)}^{i}\right)$ and $\underline{F}^{P\left(B_{1}\right)}\left(p_{B_{1}}\right)=\underline{P}\left[P\left(B_{1}\right)<p_{B_{1}}\right]=\sum_{A_{P\left(B_{1}\right)}^{i} \subset\left[0, p_{B_{1}}\right]} m\left(A_{P\left(B_{1}\right)}^{i}\right) ;$ in the same way, $\bar{F}^{P\left(B_{2}\right)}\left(p_{B_{2}}\right)=$ $\bar{P}\left[P\left(B_{2}\right)<p_{B_{2}}\right]=\sum_{A_{P\left(B_{2}\right)}^{j} \cap\left\{0, p_{B_{2}}\right] \neq 0} m\left(A_{P\left(B_{2}\right)}^{j}\right) \quad$ and $\quad \underline{F}^{P\left(B_{2}\right)}\left(p_{B_{2}}\right)=\underline{P}\left[P\left(B_{2}\right)<p_{B_{2}}\right]=$ $\sum_{A_{P\left(B_{2}\right)}^{j} \subset\left[0, p_{B_{2}}\right]} m\left(A_{P\left(B_{2}\right)}^{j}\right)$. The upper and lower CDFs, $\bar{F}^{P\left(B_{1}\right)}, \bar{F}^{P\left(B_{2}\right)}, \underline{F}^{P\left(B_{1}\right)}$ and $\underline{F}^{P\left(B_{2}\right)}$, respectively, corresponding to the illustrative DS structures $\{([0.20,0.50], 0.35)$, $([0.40,0.60], 0.65)\}$ and $\{([0.10,0.35], 0.45),([0.30,0.45], 0.55)\}$ of $P\left(B_{1}\right)$ and $P\left(B_{2}\right)$ are pictorially shown in Figure 2, bottom left and right, respectively. For example, referring again to event $B_{1}$, the upper and lower CDFs, $\bar{F}^{P\left(B_{1}\right)}$ and $\underline{F}^{P\left(B_{1}\right)}$, can be interpreted as follows: the probability $P\left[P\left(B_{1}\right)<p_{B_{1}}\right]$ that $P\left(B_{1}\right)$ is lower than or equal to, e.g., $p_{B_{1}}=0.30$ lies within interval $\left[\underline{F}^{P\left(B_{1}\right)}(0.30), \bar{F}^{P\left(B_{1}\right)}(0.30)\right]=[0,0.35]$ (referring 
to the concept of Bayesian subjective probabilities, the bounds $\left[\underline{F}^{P\left(B_{1}\right)}(0.30), \bar{F}^{P\left(B_{1}\right)}(0.30)\right]=[0,0.35]$ reflect that the analyst is not able or willing to precisely assign his/her probability $\left.P\left[P\left(B_{1}\right)<p_{B_{1}}\right]\right)$. Further details about DS structures (and DS theory of evidence) are not given here for brevity: the interested reader is referred to the copious literature in the field ${ }^{2(37-44)}$.
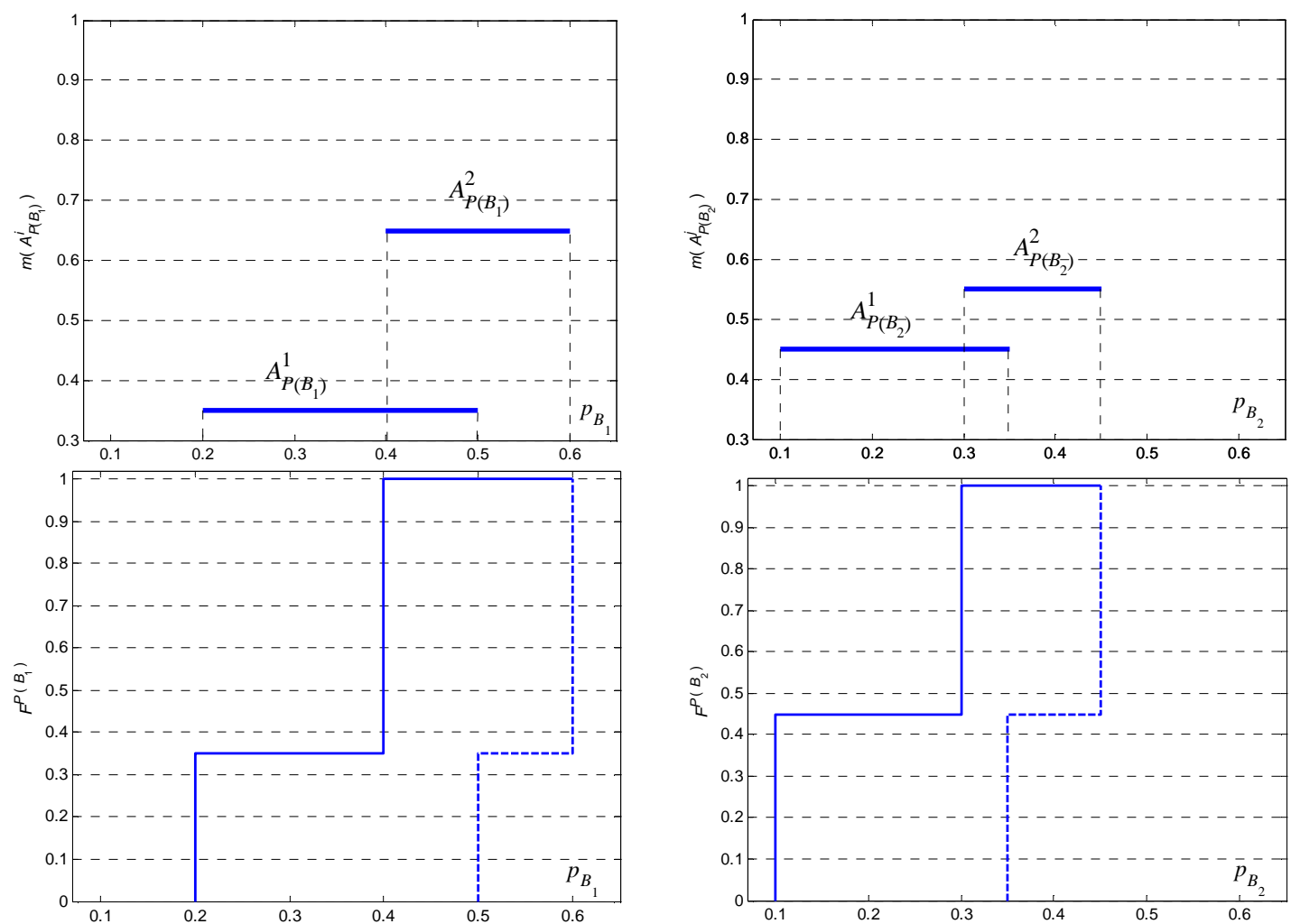

Figure 2. Top: illustrative DS structures $\{([0.20,0.50], 0.35),([0.40,0.60], 0.65)\}$ and $\{([0.10,0.35], 0.45),([0.30,0.45], 0.55)\}$ for $P\left(B_{1}\right)$ (left) and $P\left(B_{2}\right)$ (right), respectively. Bottom: upper (solid line) and lower (dashed line) CDFs, $\bar{F}^{P\left(B_{1}\right)}, \bar{F}^{P\left(B_{2}\right)}, \underline{F}^{P\left(B_{1}\right)}$ and $\underline{F}^{P\left(B_{2}\right)}$, respectively, corresponding to the illustrative DS structures described above

The focal elements $A_{P(Z)}^{i j}=\left[\underline{p}_{Z}^{i j}, \bar{p}_{Z}^{i j}\right], i=1,2, \ldots, n_{B_{1}}, j=1,2, \ldots, n_{B_{2}}$, of the probability (chance) $P(Z)$ of the event $Z=\left(B_{1} \circ_{o b j} B_{2}\right)$ are obtained as images of the focal sets $A_{P\left(B_{1}\right)}^{i}, i=$

\footnotetext{
${ }^{2}$ Notice that representing the epistemic uncertainty in the probabilities (chances) $P\left(B_{1}\right)$ and $P\left(B_{2}\right)$ by DS structures does not impair the generality of the description. Actually, any other type of distribution that may be used to describe the epistemic uncertainty in $P\left(B_{1}\right)$ and $P\left(B_{2}\right)$ can be easily transformed into a DS structure: approaches for transforming probability distributions can be found in ${ }^{(80)}$ and ${ }^{(81)}$, whereas techniques for transforming possibility distributions can be found in ${ }^{(30)}$.
} 
$1,2, \ldots, n_{B_{1}}$, and $A_{P\left(B_{2}\right)}^{j}, j=1,2, \ldots, n_{B_{2}}$, through the function $g_{Z}\left(P\left(B_{1}\right), P\left(B_{2}\right)\right)$ as $A_{P(Z)}^{i j}=$ $\left[\underline{p}_{Z}^{i j}, \bar{p}_{Z}^{i j}\right]=\left[\min _{\left.P\left(B_{1}\right) \in A_{P\left(B_{1}\right)}^{i}\right) P\left(B_{2}\right) \in A_{P\left(B_{2}\right)}^{j}}\left\{g_{Z}\left(P\left(B_{1}\right), P\left(B_{2}\right)\right)\right\}, \max _{P\left(B_{1}\right) \in A_{P\left(B_{1}\right)}^{i}, P\left(B_{2}\right) \in A_{P\left(B_{2}\right)}^{j}}\left\{g_{Z}\left(P\left(B_{1}\right), P\left(B_{2}\right)\right)\right\}\right], i=1$, $2, \ldots, n_{B_{1}}, j=1,2, \ldots, n_{B_{2}}$. For illustration purposes, again let $B_{1}$ and $B_{2}$ be the events of failure of Components 1 and 2, respectively, of the simple parallel system of Figure 1 left, and $P\left(B_{1}\right)$ and $P\left(B_{2}\right)$ the corresponding probabilities (chances): then, the probability (chance) $P(Z)$ of failure of the parallel system of Figure 1 left is the probability (chance) of the conjunction $Z=\left(B_{1} \cap_{o b j} B_{2}\right)$ of $B_{1}$ and $B_{2}$. For the sake of simplicity, we also suppose that $B_{1}$ and $B_{2}$ are (objectively) independent events (i.e., 'obj' = 'ind'): in such a case, $P(Z)$ is given by the product of $P\left(B_{1}\right)$ and $P\left(B_{2}\right)$, i.e., $P(Z)=g_{Z}\left(P\left(B_{1}\right), P\left(B_{2}\right)\right)=P\left(B_{1}\right) \cdot P\left(B_{2}\right)$ (see (1)). Finally, we suppose that $P\left(B_{1}\right)$ and $P\left(B_{2}\right)$ are distributed as in Figure 2. In this case, the lower (resp., upper) bound $\underline{p}_{Z}^{i j}$ (resp., $\bar{p}_{Z}^{i j}$ ) of the focal set $A_{P(Z)}^{i j}$ is computed as the product of the lower bounds $\underline{p}_{B_{1}}^{i}$ and $\underline{p}_{B_{2}}^{j}$ (resp., upper bounds $\bar{p}_{B_{1}}^{i}$ and $\bar{p}_{B_{2}}^{j}$ ) of the focal sets $A_{P\left(B_{1}\right)}^{i}$ and $A_{P\left(B_{2}\right)}^{j}$, respectively, i.e., $\underline{p}_{Z}^{i j}=\underline{p}_{B_{1}}^{i} \cdot \underline{p}_{B_{2}}^{j}$ (resp., $\bar{p}_{Z}^{i j}=\bar{p}_{B_{1}}^{i} \cdot \bar{p}_{B_{2}}^{j}$ ), $i=1,2, j=1,2$. Thus, it is found that $A_{P(Z)}^{11}=[0.2 \cdot 0.1,0.5 \cdot 0.35]=[0.02,0.175], A_{P(Z)}^{12}=[0.2 \cdot 0.3,0.5 \cdot 0.45]=[0.06,0.225], A_{P(Z)}^{21}=$ $[0.4 \cdot 0.1,0.6 \cdot 0.35]=[0.04,0.210], A_{P(Z)}^{22}=[0.4 \cdot 0.3,0.6 \cdot 0.45]=[0.120,0.270]$

The probability masses $m\left(A_{P(Z)}^{i j}\right)$ of the focal elements $A_{P(Z)}^{i j}, i=1,2, \ldots, n_{B_{1}}, j=1,2, \ldots$, $n_{B_{2}}$, thereby obtained have to be determined based on the state of epistemic dependence between the estimates of $P\left(B_{1}\right)$ and $P\left(B_{2}\right)$. Three conditions of epistemic dependence are often encountered in risk assessment problems and, thus, considered in this paper: i) 
independence (Section 2.2.1), ii) total (perfect) dependence (Section 2.2.2) and iii) unknown dependence $^{3}$ (Section 2.2.3).

\subsubsection{Independence}

If the distributions describing the epistemic uncertainty associated to $P\left(B_{1}\right)$ and $P\left(B_{2}\right)$ are built using different information sources (e.g., different experts, observers or data sets), then state-of-knowledge independence (item i. above, 'epi' = 'ind') exists between the estimates of $P\left(B_{1}\right)$ and $P\left(B_{2}\right)$ : in this paper, such condition is modeled by assuming random set independence between the focal elements $A_{P\left(B_{1}\right)}^{i}=\left[\underline{p}_{B_{1}}^{i}, \bar{p}_{B_{1}}^{i}\right], i=1,2, \ldots, n_{B_{1}}$, and $A_{P\left(B_{2}\right)}^{j}=\left[\underline{p}_{B_{2}}^{j}, \bar{p}_{B_{2}}^{j}\right], j=1,2, \ldots, n_{B_{2}}{ }^{(40,82-84)}$. In practice, this amounts to computing the probability masses $m\left(A_{P(Z)}^{i j}\right)$ of the focal elements $A_{P(Z)}^{i j}$ as the product of the probability masses $m\left(A_{P\left(B_{1}\right)}^{i}\right)$ and $m\left(A_{P\left(B_{2}\right)}^{j}\right)$, i.e., $m\left(A_{P(Z)}^{i j}\right)=m\left(A_{P\left(B_{1}\right)}^{i}\right) \cdot m\left(A_{P\left(B_{2}\right)}^{j}\right), i=1,2, \ldots, n_{B_{1}}, j=1,2$, $\ldots, n_{B_{2}}$. Thus, referring again to the example above, it is found that under the assumption of random set independence ('epi' = 'ind') the probability masses of the focal sets $A_{P(Z)}^{11}=[0.02$, $0.175], A_{P(Z)}^{12}=[0.06,0.225], A_{P(Z)}^{21}=[0.04,0.210]$ and $A_{P(Z)}^{22}=[0.120,0.270]$ are $m\left(A_{P(Z)}^{11}\right)=$ $0.35 \cdot 0.45=0.1575, m\left(A_{P(Z)}^{12}\right)=0.35 \cdot 0.55=0.1925, m\left(A_{P(Z)}^{21}\right)=0.65 \cdot 0.45=0.2925$ and $m\left(A_{P(Z)}^{22}\right)=0.65 \cdot 0.55=0.3575$, respectively. The corresponding upper and lower CDFs, $\bar{F}^{P(Z)}$ and $\underline{F}^{P(Z)}$, of the probability (chance) $P(Z)$ of $Z=\left(B_{1} \cap_{\text {ind }} B_{2}\right)^{\text {ind }}$ are shown in Figure 3, top left.

\footnotetext{
${ }^{3}$ In the rest of the paper, the state of epistemic dependence between the probabilities (chances) $P\left(B_{1}\right)$ and $P\left(B_{2}\right)$ of events $B_{1}$ and $B_{2}$ linked to an event $Z$ of interest by the logical connection ' ${ }_{o b j}$ ' is indicated as $\left(B_{1} \circ_{o b j} B_{2}\right)^{e p i}$, where the superscript 'epi' stands for 'ind', 'perf' or 'ukn' in the cases of independence, total (perfect) or unknown epistemic dependence, respectively.
} 


\subsubsection{Total (perfect) dependence}

When the same information source is employed to construct the uncertainty distributions for $P\left(B_{1}\right)$ and $P\left(B_{2}\right)$, then total (perfect) dependence (item ii. above, 'epi' = 'perf') exists between the estimates of $P\left(B_{1}\right)$ and $P\left(B_{2}\right)^{(2,11)}$. By way of example, consider the case of a system containing a number of physically distinct, but similar/nominally identical components whose failure probabilities (chances) are estimated by means of the same data set: in such situation, the state of knowledge about these failure probabilities (chances) is exactly the same and, thus, the distributions describing the epistemic uncertainty associated to such failure probabilities (chances) have to be considered totally (perfectly) dependent ${ }^{4}(2,11)$. In this paper, such condition is straightforwardly modeled by imposing maximal correlation between the distributions of $P\left(B_{1}\right)$ and $P\left(B_{2}\right)^{(2,11)}$. In practice, assuming that the distributions of $P\left(B_{1}\right)$ and $P\left(B_{2}\right)$ are totally (perfectly) correlated implies that when one uncertain parameter (e.g., $\left.P\left(B_{1}\right)\right)$ is large with reference to its statistical distribution, then also the other uncertain parameter (e.g., $\left.P\left(B_{2}\right)\right)$ is large "to the same degree with respect to its own statistical distribution" ${ }^{(40)}$. This "empirical" definition suggests the computational strategy for simulating total (perfect) correlation between the distributions of the uncertain parameters $P\left(B_{1}\right)$ and $P\left(B_{2}\right):$ i) choose a set of $n_{B}$ (equally spaced) values $\beta^{i}, i=1,2, \ldots, n_{B}$, within $[0,1)$ (e.g., $\beta^{1}=0, \beta^{2}=0.01, \ldots, \beta^{n B-1}=0.99, \beta^{n B}=1$ ); ii) identify the corresponding focal sets $A_{P\left(B_{1}\right)}^{i}=\left[\underline{p}_{B_{1}}^{i}, \bar{p}_{B_{1}}^{i}\right]$ and $A_{P\left(B_{2}\right)}^{i}=\left[\underline{p}_{B_{2}}^{i}, \bar{p}_{B_{2}}^{i}\right]$ of $P\left(B_{1}\right)$ and $P\left(B_{2}\right)$ using the inverse transform

\footnotetext{
${ }^{4}$ As stated in Ref. 2-Page 54, "an analyst's state of knowledge about the possible values of a parameter $\theta$ can be expressed in terms of a probability distribution $f^{\theta}(\theta)$ when using Bayesian updating or expert judgment. It is common practice to assign the same value to the parameters of BEs of identical or similar components. Therefore, for example, the probability of failure of a class of identical motor-operated valves (MOVs) to open is considered the same. Suppose that $\theta_{1}$ and $\theta_{2}$ represent the parameters of two physically distinct but identical MOVs: because this discussion assumes that all such MOVs have the same parameter, it is necessary to set $\theta_{1}=$ $\theta_{2}$. Moreover, because the analyst's state of knowledge is the same for the two valves, it follows that $f^{\theta_{1}}\left(\theta_{1}\right)=$ $f^{\theta_{2}}\left(\theta_{2}\right)$. Thus, $f^{\theta_{1}}\left(\theta_{1}\right)$ and $f^{\theta_{2}}\left(\theta_{2}\right)$ must be regarded as being equal distributions and treated as completely dependent distributions".
} 
method, i.e., $\left[\left(\bar{F}^{P\left(B_{1}\right)}\right)^{-1}\left(\beta^{i}\right),\left(\underline{F}^{P\left(B_{1}\right)}\right)^{-1}\left(\beta^{i}\right)\right]$ and $\left[\left(\bar{F}^{P\left(B_{2}\right)}\right)^{-1}\left(\beta^{i}\right),\left(\underline{F}^{P\left(B_{2}\right)}\right)^{-1}\left(\beta^{i}\right)\right], i=1,2, \ldots, n_{B}$, respectively (notice that using the same values $\beta^{i}$ for the identification of the focal sets of both $P\left(B_{1}\right)$ and $P\left(B_{2}\right)$ implies total (perfect) dependence between them) ${ }^{(11)}$; iii) calculate the focal elements $A_{P(Z)}^{i}$ as $\left[\min _{P\left(B_{1}\right) \in A_{P\left(B_{1}\right)}^{i}, P\left(B_{2}\right) \in A_{P\left(B_{2}\right)}^{i}}\left\{g_{Z}\left(P\left(B_{1}\right), P\left(B_{2}\right)\right)\right\}, \max _{P\left(B_{1}\right) \in A_{P\left(B_{1}\right)}^{i}, P\left(B_{2}\right) \in A_{P\left(B_{2}\right)}^{i}}\left\{g_{Z}\left(P\left(B_{1}\right), P\left(B_{2}\right)\right)\right\}\right]$, $i=1,2, \ldots, n_{B} ;$ iv) associate to $A_{P(Z)}^{i}$ the probability mass $m\left(A_{P(Z)}^{i}\right)=1 / n_{B}, i=1,2, \ldots, n_{B}$. Referring again to the example above, it is found that under the assumption of total (perfect) epistemic dependence the probability masses of the focal sets $A_{P(Z)}^{11}=[0.02,0.175], A_{P(Z)}^{12}=$ $[0.06,0.225], A_{P(Z)}^{21}=[0.04,0.210]$ and $A_{P(Z)}^{22}=[0.120,0.270]$ obtained by performing steps i)-iv) above are $m\left(A_{P(Z)}^{11}\right)=0.35, m\left(A_{P(Z)}^{12}\right)=0, m\left(A_{P(Z)}^{21}\right)=0.10$ and $m\left(A_{P(Z)}^{22}\right)=0.55$, respectively. The resulting upper and lower CDFs, $\bar{F}^{P(z)}$ and $\underline{F}^{P(z)}$, of the probability (chance) $P(Z)$ of $Z=\left(B_{1} \cap_{\text {ind }} B_{2}\right)^{\text {perf }}$ are shown in Figure 3, top right.

\subsubsection{Unknown dependence}

When the state of dependence between the information sources used to build the distributions of $P\left(B_{1}\right)$ and $P\left(B_{2}\right)$ cannot be defined precisely by the analyst (item iii. above, 'epi' = 'ukn'), for the sake of conservatism all kinds of (possibly unknown) epistemic dependences between the estimates of $P\left(B_{1}\right)$ and $P\left(B_{2}\right)$ have to be accounted for. In this paper, the Distribution Envelope Determination (DEnv) method ${ }^{(61-65)}$ is adopted to this aim. The DEnv method allows computing extreme upper and lower Cumulative Distribution Functions (CDFs) $\bar{F}_{D E n v}^{P(Z)}\left(p_{Z}\right)$ and $\underline{F}_{D E n v}^{P(Z)}\left(p_{Z}\right)$ on the probability (chance) $P(Z)=g_{Z}\left(P\left(B_{1}\right), P\left(B_{2}\right)\right)$ of the event $Z$ $=\left(B_{1} \circ_{o b j} B_{2}\right)^{u k n}$ of interest no matter what correlations or dependencies exist among $P\left(B_{1}\right)$ and $P\left(B_{2}\right)$; these bounds are also the "pointwise best possible, which means they could not be any tighter without excluding some possible dependences" ${ }^{(40)}$. In practice, the aim of the 
DEnv approach is to identify the $n_{B_{1}} \cdot n_{B_{2}}$ probability masses $m\left(A_{P(Z)}^{i j}\right)$ for the focal elements $A_{P(Z)}^{i j}, i=1,2, \ldots, n_{B_{1}}, j=1,2, \ldots, n_{B_{2}}$, such that the upper CDF on $P(Z)$ is the maximal possible (i.e., $\bar{F}_{D E n v}^{P(Z)}\left(p_{Z}\right)=\max \left\{\bar{F}^{P(Z)}\left(p_{Z}\right)\right\}$ ) and the lower CDF on $P(Z)$ is the minimal possible $\left(\underline{F}_{D E n v}^{P(Z)}\left(p_{Z}\right)=\min \left\{\underline{F}^{P(Z)}\left(p_{Z}\right)\right\}\right)$ provided that a precise set of constraints is satisfied ${ }^{(61-65)}$. In more detail, $\bar{F}_{D E n v}^{P(Z)}\left(p_{Z}\right)$ and $\underline{F}_{D E n v}^{P(Z)}\left(p_{Z}\right)$ are found by solving the following linear maximization (13) and minimization (14) problems, respectively:

Find $m\left(A_{P(Z)}^{i j}\right), i=1,2, \ldots, n_{B_{1}}, j=1,2, \ldots, n_{B_{2}}$ :

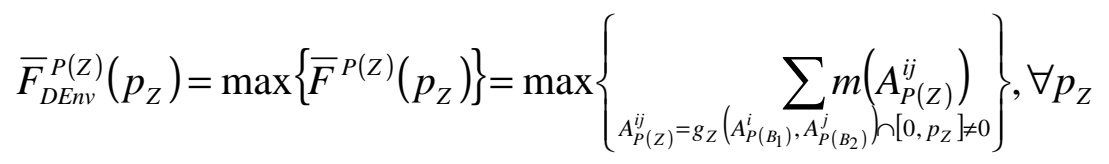

Find $m\left(A_{P(Z)}^{i j}\right), i=1,2, \ldots, n_{B_{1}}, j=1,2, \ldots, n_{B_{2}}$ :

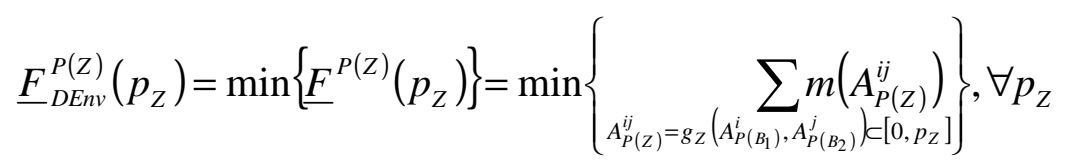

subject to the constraints that i) the probability masses $m\left(A_{P\left(B_{1}\right)}^{i}\right)$ and $m\left(A_{P\left(B_{2}\right)}^{j}\right)$ are conserved (i.e., $\sum_{i=1}^{n_{B_{1}}} m\left(A_{P(Z)}^{i j}\right)=m\left(A_{P\left(B_{2}\right)}^{j}\right), j=1,2, \ldots, n_{B_{2}}$, and $\left.\sum_{j=1}^{n_{B_{2}}} m\left(A_{P(Z)}^{i j}\right)=m\left(A_{P\left(B_{1}\right)}^{i}\right), i=1,2, \ldots, n_{B_{1}}\right)$ and ii) the probability masses $m\left(A_{P(Z)}^{i j}\right)$ are larger than or equal to zero. For illustration purposes, the values of $\bar{F}_{D E n v}^{P(Z)}\left(p_{Z}\right)=\bar{F}_{D E n v}^{P(Z)}(0.08)$ and $\underline{F}_{D E n v}^{P(Z)}\left(p_{Z}\right)=\underline{F}_{D E n v}^{P(Z)}(0.22)$ are calculated with reference to the example above. In order to calculate $\bar{F}_{D E n v}^{P(Z)}(0.08)$ by solving maximization problem (13), those focal sets among $A_{P(Z)}^{i j}, i=1,2, . j=1,2$, that intersect interval $\left[0, p_{Z}\right]=[0,0.08]$ have to be identified. Since in this case $A_{P(Z)}^{11}=[0.02,0.175], A_{P(Z)}^{12}$ $=[0.06,0.225], A_{P(Z)}^{21}=[0.04,0.210]$ and $A_{P(Z)}^{22}=[0.120,0.270]$ (see above), only focal sets $A_{P(Z)}^{11}, A_{P(Z)}^{12}$ and $A_{P(Z)}^{21}$ intersect interval [0, 0.08]; then, only focal sets $A_{P(Z)}^{11}, A_{P(Z)}^{12}$ and $A_{P(Z)}^{21}$ and the corresponding probability masses $m\left(A_{P(Z)}^{11}\right), m\left(A_{P(Z)}^{12}\right)$ and $m\left(A_{P(Z)}^{21}\right)$ have to be 
included in the function $\bar{F}^{P(Z)}(0.08)$ to be maximized. As a consequence, maximization problem (13) becomes:

Find $m\left(A_{P(Z)}^{11}\right), m\left(A_{P(Z)}^{12}\right), m\left(A_{P(Z)}^{21}\right), m\left(A_{P(Z)}^{22}\right)$ :

$$
\bar{F}_{D E n v}^{P(Z)}(0.08)=\max \left\{\bar{F}^{P(Z)}(0.08)\right\}=\max \left\{m\left(A_{P(Z)}^{11}\right)+m\left(A_{P(Z)}^{12}\right)+m\left(A_{P(Z)}^{21}\right)\right\}
$$

subject to the constraints that i) $m\left(A_{P(Z)}^{11}\right)+m\left(A_{P(Z)}^{12}\right)=m\left(A_{P\left(B_{1}\right)}^{1}\right)=0.35, m\left(A_{P(Z)}^{21}\right)+m\left(A_{P(Z)}^{22}\right)=$ $m\left(A_{P\left(B_{1}\right)}^{2}\right)=0.65, m\left(A_{P(Z)}^{11}\right)+m\left(A_{P(Z)}^{21}\right)=m\left(A_{P\left(B_{2}\right)}^{1}\right)=0.45, m\left(A_{P(Z)}^{12}\right)+m\left(A_{P(Z)}^{22}\right)=m\left(A_{P\left(B_{2}\right)}^{2}\right)=$ 0.55 and ii) $m\left(A_{P(Z)}^{11}\right), m\left(A_{P(Z)}^{12}\right), m\left(A_{P(Z)}^{21}\right), m\left(A_{P(Z)}^{22}\right) \geq 0$. The optimization process leads to $\bar{F}_{D E n v}^{P(Z)}(0.08)=0.8$ with $m\left(A_{P(Z)}^{11}\right)=0, m\left(A_{P(Z)}^{12}\right)=0.35, m\left(A_{P(Z)}^{21}\right)=0.45$ and $m\left(A_{P(Z)}^{22}\right)=0.2$.

Instead, in order to calculate $\underline{F}_{D E n v}^{P(Z)}(0.22)$ by solving minimization problem (14), those focal sets among $A_{P(Z)}^{i j}, i=1,2, . j=1,2$, that are included in interval $\left[0, p_{Z}\right]=[0,0.22]$ have to be identified. Since in this case $A_{P(Z)}^{11}=[0.02,0.175], A_{P(Z)}^{12}=[0.06,0.225], A_{P(Z)}^{21}=[0.04,0.210]$ and $A_{P(Z)}^{22}=[0.120,0.270]$ (see above), only focal sets $A_{P(Z)}^{11}$ and $A_{P(Z)}^{21}$ are included in interval [0, 0.22]; then, only $A_{P(Z)}^{11}$ and $A_{P(Z)}^{21}$ and the corresponding probability masses $m\left(A_{P(Z)}^{11}\right)$ and $m\left(A_{P(Z)}^{21}\right)$ have to be taken into account in the function $\underline{F}^{P(Z)}(0.22)$ to be minimized. Then, minimization problem (14) becomes:

Find $m\left(A_{P(Z)}^{11}\right), m\left(A_{P(Z)}^{12}\right), m\left(A_{P(Z)}^{21}\right), m\left(A_{P(Z)}^{22}\right)$ :

$$
\underline{F}_{D E n v}^{P(Z)}(0.22)=\min \left\{F^{P(Z)}(0.22)\right\}=\min \left\{m\left(A_{P(Z)}^{11}\right)+m\left(A_{P(Z)}^{21}\right)\right\}
$$

subject to the same constraints as (15). The optimization process leads to $\underline{F}_{D E n v}^{P(Z)}(0.22)=0.45$ with $m\left(A_{P(Z)}^{11}\right)=0.15, m\left(A_{P(Z)}^{12}\right)=0.20, m\left(A_{P(Z)}^{21}\right)=0.30$ and $m\left(A_{P(Z)}^{22}\right)=0.35$.

Finally, it is worth noting that in order to construct the entire $\operatorname{CDFs} \bar{F}_{D E n v}^{P(Z)}\left(p_{Z}\right)$ and $\underline{F}_{D E n v}^{P(Z)}\left(p_{Z}\right)$ for $P(Z)$, such optimization problems have to be solved for all the values $p_{Z}$ of interest. The resulting upper and lower CDFs, $\bar{F}^{P(Z)}$ and $\underline{F}^{P(Z)}$, of the probability (chance) $P(Z)$ of $Z=\left(B_{1}\right.$ $\left.\cap_{\text {ind }} B_{2}\right)^{u k n}$ are shown in Figure 3, bottom. 

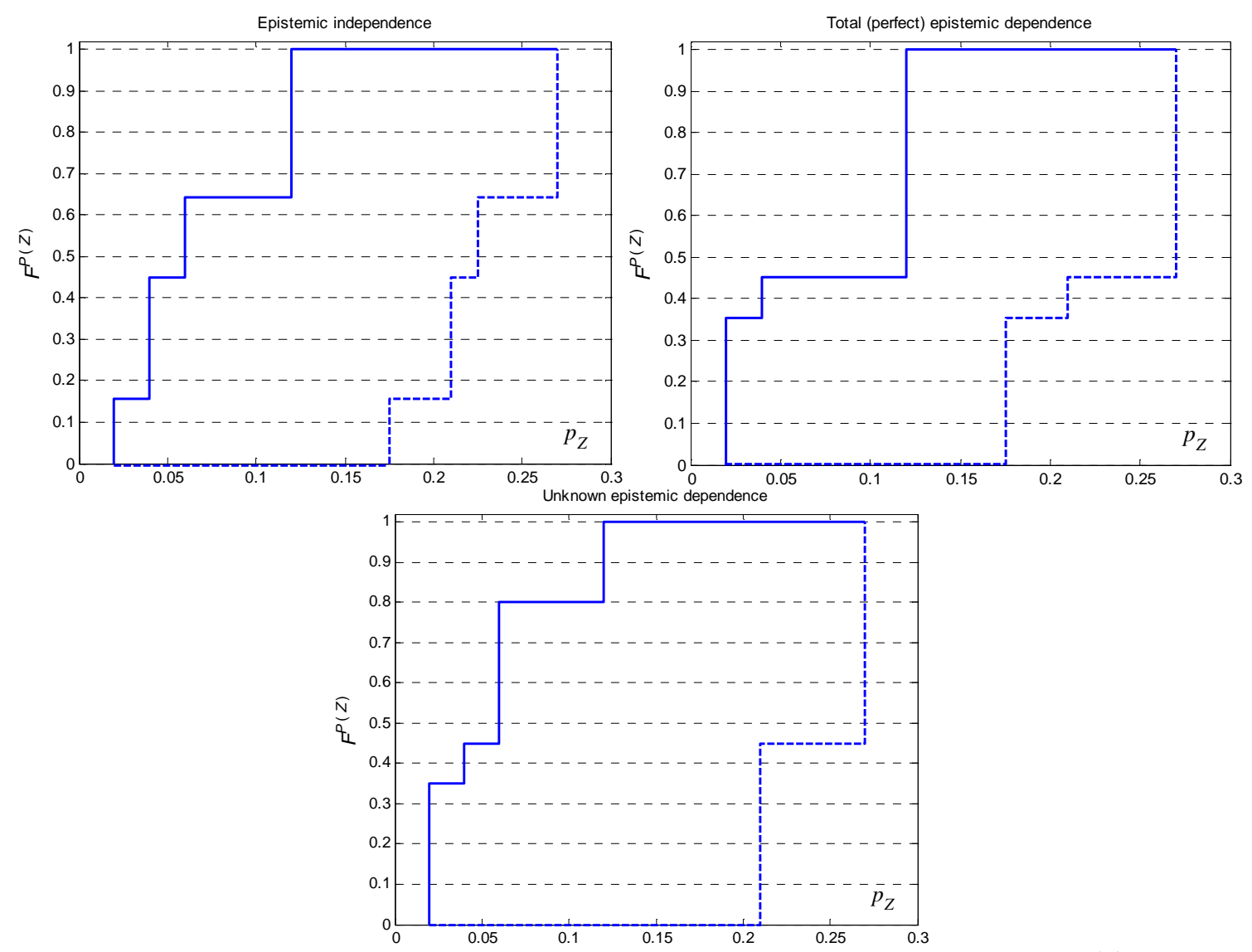

Figure 3. Upper (solid lines) and lower (dashed lines) CDFs, $\bar{F}^{P(Z)}$ and $\underline{F}^{P(Z)}$, of the probability (chance) $P(Z)$ of the conjunction of two (objectively) independent events $B_{1}$ and $B_{2}$ with probabilities (chances) $P\left(B_{1}\right)$ and $P\left(B_{2}\right)$ distributed as in Figure 2, under the assumptions of independence (top left), total (top right) and unknown (bottom) epistemic dependence

\section{CASE STUDY}

In this Section, we present the example FT used for reference. In Section 3.1, the FT structure and BEs uncertainties are described; in Section 3.2, the different states of (objective and epistemic) dependence between the BEs are summarized; in Section 3.3, the numerical indicators used to quantify the effects of such dependences are provided.

\subsection{Fault tree structure and basic events uncertainties}

A simple FT comprised of $n_{B E}=6$ BEs $\left\{B_{i}: i=1,2, \ldots, n_{B E}=6\right\}$ is considered (Figure 4). BEs $B_{1}, B_{2}$ and $B_{3}$ are linked to event $E_{1}$ by junction $J_{1}$ (an OR-gate) and BEs $B_{4}, B_{5}$ and $B_{6}$ 
are linked to event $E_{2}$ by junction $J_{2}$ (also an OR-gate); finally, events $E_{1}$ and $E_{2}$ are linked to the Top Event (TE) $X$ by junction $J_{3}$ (an AND-gate):

$$
X=E_{1} \cap E_{2}=\left(B_{1} \cup B_{2} \cup B_{3}\right) \cap\left(B_{4} \cup B_{5} \cup B_{6}\right)
$$

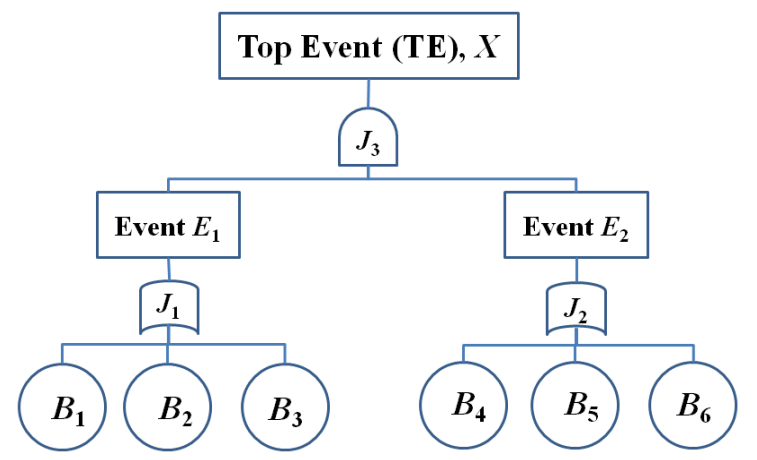

Figure 4. FT structure

Letting $\left\{P\left(B_{i}\right): i=1,2, \ldots, n_{B E}=6\right\}$ denote the probabilities (chances) of BEs $\left\{B_{i}: i=1,2, \ldots\right.$, $\left.n_{B E}=6\right\}$, the probability (chance) $P(X)$ of the TE $X$ is expressed in all generality as follows:

$P(X)=g_{X}\left(P\left(B_{1}\right), P\left(B_{2}\right), P\left(B_{3}\right), P\left(B_{4}\right), P\left(B_{5}\right), P\left(B_{6}\right)\right)$,

where $g_{X}(\cdot)$ is a deterministic function of i) the FT structure (i.e., the logical connections between the BEs) (see Figure 4) and ii) the (possible) objective dependences existing between the BEs (see Sections 2 and 3.2).

It is assumed that $\left\{P\left(B_{i}\right): i=1,2, \ldots, n_{B E}=6\right\}$ are epistemically-uncertain. Uncertainties about $\left\{P\left(B_{i}\right): i=1,6\right\}$ are described using lognormal Probability Distribution Functions (PDFs) $\left\{f^{P\left(B_{i}\right)}\left(p_{B_{i}}\right)=L N\left(\mu_{i}, \sigma_{i}\right): i=1,6\right\}$ with parameter values $\left\{\left(\mu_{i}, \sigma_{i}\right): i=1,6\right\}$ as specified in Table I. As an example, $B_{1}$ and $B_{6}$ could denote failure of an item (e.g., a mechanical component) for which a sufficient amount of informative (failure) data is available for statistical analysis and for accurate characterization of the corresponding epistemic uncertainty by a precise probability distribution. Differently, uncertainties about $\left\{P\left(B_{i}\right): i=2,3,5\right\}$ are represented using (trapezoidal) possibility distributions $\left\{\pi^{P\left(B_{i}\right)}\left(p_{B_{i}}\right)=\operatorname{TRAP}\left(a_{i}, b_{i}, c_{i}, d_{i}\right): i=2,3,5\right\}$, with supports $\left\{\left[a_{i}, d_{i}\right]: i=2,3,5\right\}$ and cores $\left\{\left[b_{i}\right.\right.$, 
$\left.\left.c_{i}\right]: i=2,3,5\right\}$ as specified in Table I. By way of example, $B_{2}, B_{3}$ and $B_{5}$ could denote events (e.g., human-error-dominated events) for which no data exists and where the (trapezoidal) possibility distributions are constructed based on expert statements alone. Finally, the uncertainty about $P\left(B_{4}\right)$ is described by a finite Dempster-Shafer (DS) structure, i.e., by a set of $n_{B_{4}}=4$ intervals (focal elements) $A_{P\left(B_{4}\right)}^{j}=\left[\underline{p}_{B_{4}}^{j}, \bar{p}_{B_{4}}^{j}\right], j=1,2, \ldots, n_{B_{4}}=4$, each of which is assigned a probability mass $m\left(A_{P\left(B_{4}\right)}^{j}\right), j=1,2, \ldots, n_{B_{4}}=4$, as specified in Table I. As an example, $B_{4}$ could denote failure of an item (e.g., a protective or automation system, a digital instrumentation and control system, a recently-developed technology, ...) for which only sparse pieces of data exist: in such cases, the available information is much more valuable than purely subjective (and often vague) expert judgment, but it is not sufficient for building a precise probability distribution.

Two different cases are considered: 'large' (Case A) and 'small' (Case B) BE probabilities (chances). In Case A, $\left\{P\left(B_{i}\right): i=1,2, \ldots, n_{B E}=6\right\}$ are of the order of $10^{-1}$, whereas in Case B they are of the order of $10^{-3}$ (Table I). For illustration purposes, Figure 5 shows the distributions of $\left\{P\left(B_{i}\right): i=1,2, \ldots, n_{B E}=6\right\}$, with reference only to Case B.

\begin{tabular}{lll}
\hline $\boldsymbol{P}\left(\boldsymbol{B}_{1}\right)$ & & \\
\hline Epistemic uncertainty description & Probability distribution \\
\hline Distribution shape & & Lognormal, $f^{P\left(B_{1}\right)}\left(p_{B_{1}}\right)=L N\left(\mu_{1}, \sigma_{1}\right)$ \\
\hline $\begin{array}{l}\text { Distribution } \\
\text { parameters }\end{array}$ & Case A & $\mu_{1}=-1.6094, \sigma_{1}=0.3226$ \\
\hline $\boldsymbol{P}\left(\boldsymbol{B}_{2}\right)$ & Case B & $\mu_{1}=-5.8091, \sigma_{1}=0.6678$ \\
\hline Epistemic uncertainty description & Possibility distribution \\
\hline Distribution shape & & Trapezoidal, $\pi^{P\left(B_{2}\right)}\left(p_{B_{2}}\right)=T R A P\left(a_{2}, b_{2}, c_{2}, d_{2}\right)$ \\
\hline $\begin{array}{l}\text { Distribution } \\
\text { parameters }\end{array}$ & Case A & $a_{2}=1 \cdot 10^{-1}, b_{2}=1.5 \cdot 10^{-1}, c_{2}=2.5 \cdot 10^{-1}, d_{2}=4 \cdot 10^{-1}$ \\
\hline $\boldsymbol{P}\left(\boldsymbol{B}_{3}\right)$ & Case B & $a_{2}=2 \cdot 10^{-3}, b_{2}=3 \cdot 10^{-3}, c_{2}=5 \cdot 10^{-3}, d_{2}=8 \cdot 10^{-3}$ \\
\hline Epistemic uncertainty description & Possibility distribution \\
\hline Distribution shape & & Trapezoidal, $\pi^{P\left(B_{3}\right)}\left(p_{B_{3}}\right)=T R A P\left(a_{3}, b_{3}, c_{3}, d_{3}\right)$ \\
\hline $\begin{array}{l}\text { Distribution } \\
\text { parameters }\end{array}$ & Case A & $a_{3}=2.5 \cdot 10^{-1}, b_{3}=4 \cdot 10^{-1}, c_{3}=4 \cdot 10^{-1}, d_{3}=5 \cdot 10^{-1}$ \\
\hline $\boldsymbol{P}\left(\boldsymbol{B}_{4}\right)$ & Case B & $a_{3}=5 \cdot 10^{-3}, b_{3}=8 \cdot 10^{-3}, c_{3}=8 \cdot 10^{-3}, d_{3}=1 \cdot 10^{-2}$ \\
\hline & & \\
\hline
\end{tabular}




\begin{tabular}{lll}
\hline \multicolumn{2}{l}{ Epistemic uncertainty description } & Dempster-Shafer (DS) structure \\
\hline Distribution shape & & $\left\{\left(A_{P\left(B_{4}\right.}^{j}, m\left(A_{P\left(B_{4}\right)}^{j}\right)\right): j=1,2, \ldots, n_{B_{4}}=4\right\}$ \\
\hline \multirow{2}{*}{$\begin{array}{l}\text { Distribution } \\
\text { parameters }\end{array}$} & Case A & $\begin{array}{l}\left\{\left(\left[5 \cdot 10^{-2}, 2.5 \cdot 10^{-1}\right], 0.19\right),\left(\left[1 \cdot 10^{-1}, 1.5 \cdot 10^{-3}\right], 0.33\right),\left(\left[2.5 \cdot 10^{-1}, 4 \cdot 10^{-1}\right],\right.\right. \\
\left.0.25),\left(\left[2 \cdot 10^{-1}, 3 \cdot 10^{-1}\right], 0.23\right)\right\}\end{array}$ \\
\cline { 2 - 3 } & Case B & $\begin{array}{l}\left\{\left(\left[1 \cdot 10^{-3}, 5 \cdot 10^{-3}\right], 0.19\right),\left(\left[2 \cdot 10^{-3}, 3 \cdot 10^{-3}\right], 0.33\right),\left(\left[5 \cdot 10^{-3}, 8 \cdot 10^{-3}\right], 0.25\right),\right. \\
\left.\left(\left[4 \cdot 10^{-3}, 6 \cdot 10^{-3}\right], 0.23\right)\right\}\end{array}$ \\
\hline $\boldsymbol{P}\left(\boldsymbol{B}_{5}\right)$ & \\
\hline Epistemic uncertainty description & Possibility distribution \\
\hline Distribution shape & & Trapezoidal, $\pi^{P\left(B_{5}\right)}\left(p_{B_{5}}\right)=T R A P\left(a_{5}, b_{5}, c_{5}, d_{5}\right)$ \\
\hline $\begin{array}{l}\text { Distribution } \\
\text { parameters }\end{array}$ & Case A & $a_{5}=5 \cdot 10^{-2}, b_{5}=2 \cdot 10^{-1}, c_{5}=2 \cdot 10^{-1}, d_{5}=4.5 \cdot 10^{-3}$ \\
\hline $\boldsymbol{P}\left(\boldsymbol{B}_{\boldsymbol{6}}\right)$ & Case B & $a_{5}=1 \cdot 10^{-3}, b_{5}=4 \cdot 10^{-3}, c_{5}=4 \cdot 10^{-3}, d_{5}=9 \cdot 10^{-3}$ \\
\hline
\end{tabular}

Epistemic uncertainty description Probability distribution

Distribution shape

Distribution Lognormal, $f^{P\left(B_{6}\right)}\left(p_{B_{6}}\right)=L N\left(\mu_{6}, \sigma_{6}\right)$

parameters

Case A $\quad \mu_{6}=-1.3863, \sigma_{6}=0.2465$

Table I. Characteristics and parameters of the distributions of $\left\{P\left(B_{i}\right): i=1,2, \ldots, n_{B E}=6\right\}$
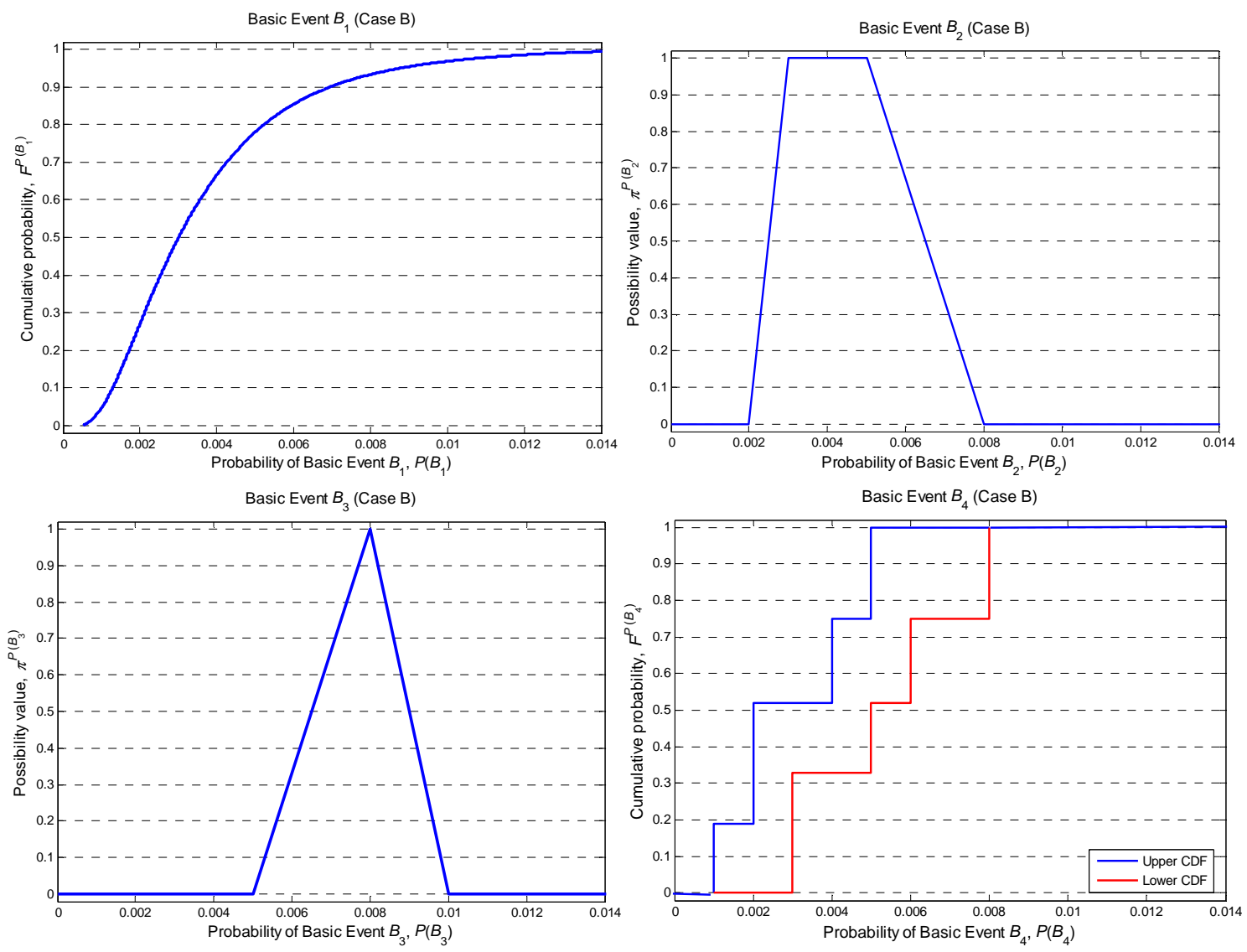

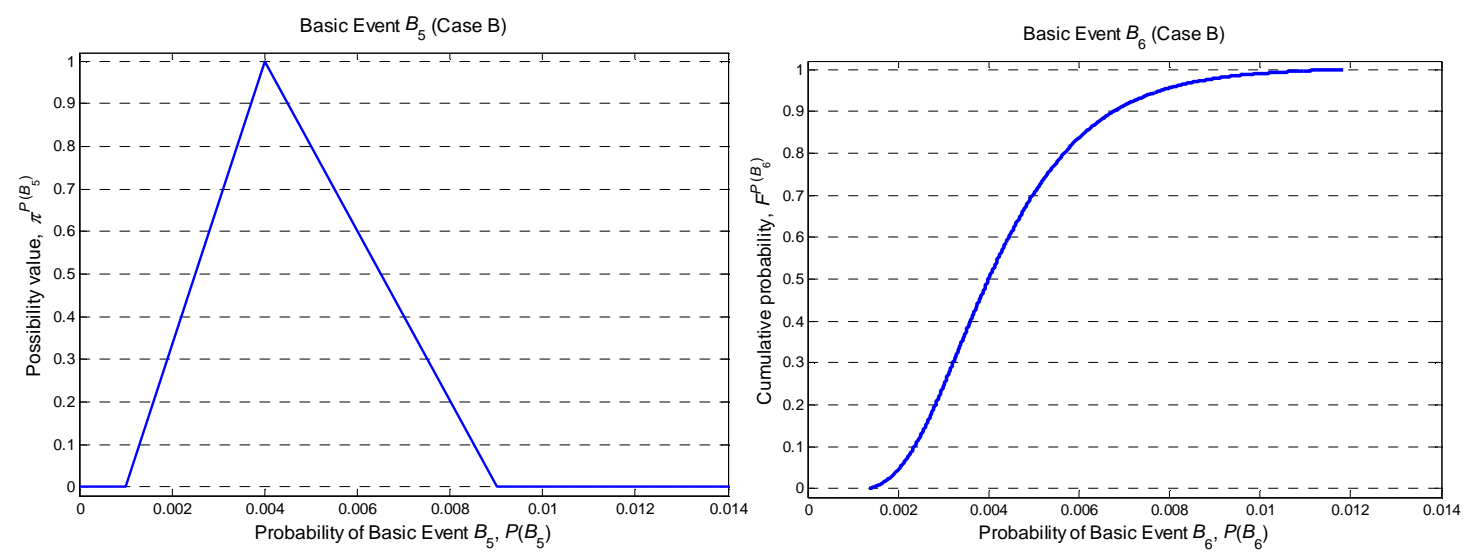

Figure 5. Distributions of $\left\{P\left(B_{i}\right): i=1,2, \ldots, n_{B E}=6\right\}$ for Case $B$

\subsection{States of dependence considered}

The following states of objective dependence between the BEs of Section 3.1 are considered in the analysis (Section 2.1): a) independence (see (1) and (2)), b) perfect (see (3) and (4)), c) opposite (see (5) and (6)), d) positive (see (9) and (10)), e) negative (see (11) and (12)) and f) unknown dependence (see (7) and (8)). In addition, the following states of epistemic dependence between the probabilities (chances) of the BEs of Section 3.1 are considered in the analysis (Section 2.2): i) independence, ii) perfect and iii) unknown dependence.

Two classes of analyses are performed (Section 4):

1. assuming unknown epistemic dependence (iii. above) between the probabilities (chances) of the BEs, the effects of different states (a.-f. above) of objective dependence between the BEs are analyzed;

2. assuming objective independence (a. above) between the BEs, the effects of different states (i.-iii. above) of epistemic dependence between the probabilities (chances) of the BEs are analyzed.

Table II summarizes the analyses carried out in the present paper (Section 4) together with the corresponding objectives.

\begin{tabular}{|c|l|l|l|}
\hline & \multicolumn{2}{|c|}{ States of dependence between the BEs } & \multicolumn{1}{c|}{ Aim of the analysis } \\
\hline & Objective (Section 2.1) & Epistemic (Section 2.2) & \multicolumn{1}{c|}{$\begin{array}{c}\text { - study the effects of different states of objective } \\
\text { dependence between the BEs of the FT when the state }\end{array}$} \\
\hline $\begin{array}{c}\text { Analysis 1 } \\
\text { (Table III }\end{array}$ & $\begin{array}{l}\text { a) independence } \\
\text { b) perfect dependence }\end{array}$ & iii) unknown dependence
\end{tabular}




\begin{tabular}{|c|l|l|l|}
\hline $\begin{array}{c}\text { and Section } \\
\text { 4.1) }\end{array}$ & $\begin{array}{l}\text { c) opposite dependence } \\
\text { d) positive dependence } \\
\text { e) negative dependence } \\
\text { f) unknown dependence }\end{array}$ & $\begin{array}{l}\text { of epistemic dependence between the probabilities } \\
\text { (chances) of the BEs is given (in particular, unknown } \\
\text { epistemic dependence is assumed in the present } \\
\text { analysis) }\end{array}$ \\
\hline $\begin{array}{c}\text { Analysis 2 } \\
\text { (Table III } \\
\text { and Section } \\
\text { 4.2) }\end{array}$ & a) independence & $\begin{array}{l}\text { - study the effects of different states of epistemic } \\
\text { dependence between the probabilities (chances) of the } \\
\text { BEs of the FT when the state of objective dependence } \\
\text { between the BEs is given (in particular, objective } \\
\text { independence is assumed in the present analysis) }\end{array}$ \\
\hline
\end{tabular}

Table II. Analyses performed in Section 4, and their relative objectives

For clarity, Table III reports the details of Analyses 1 and 2 (Table II). First, only for illustration purposes the effects of different states of (objective and epistemic) dependences between BEs $\left\{B_{i}: i=1,2, \ldots, n_{B E}=6\right\}$ are demonstrated with reference to very simple configurations (referred to as C1-C5 in Table III). In particular, events $Z=\left(B_{1} \cap B_{6}\right)(\mathrm{C} 1),\left(B_{1}\right.$ $\left.\cap B_{5}\right)(\mathrm{C} 2),\left(B_{2} \cap B_{5}\right)(\mathrm{C} 3),\left(B_{4} \cup B_{5}\right)(\mathrm{C} 4)$ and $\left(B_{2} \cup B_{3}\right)(\mathrm{C} 5)$ are considered in both Analyses 1 and 2 to study whether (and how) the effects of different states of (objective and epistemic) dependence are influenced by the particular logical connection existing between the BEs. Moreover, such analyses are performed in both Case A (namely, 'large' BE probabilities-chances) and Case B (namely, 'small' BE probabilities-chances) to study whether (and how) the effects of different states of (objective and epistemic) dependence are influenced by the magnitude of the BE probabilities (chances).

Then, the more realistic case involving the FT of Figure 4 is considered to analyze the effects that (objective and epistemic) dependences between BEs $\left\{B_{i}: i=1,2, \ldots, n_{B E}=6\right\}$ have on the probability (chance) $P(X)$ of the TE $X$ (Table III, Configurations T1-T4 of Analysis 1 and T1-T3 of Analysis 2). These computations are performed only in Case B (namely, 'small' BE probabilities-chances) because in realistic safety-critical engineered systems the basic components are usually highly reliable and, thus, the corresponding failure probabilities (chances) are typically very small. In Analysis 1, Configuration T1 represents the reference, baseline case where all the BEs are considered independent. On the opposite, Configuration T4 represents the extreme (most conservative) case where no assumptions about the states of 
objective dependence between all the BEs are made. Instead, Configurations T2 and T3 represent 'intermediate' (and more realistic) cases. In particular, in Configuration T2 positive objective dependence is assumed between BEs $B_{1}$ and $B_{6}$ (i.e., those events representing failures of mechanical components): this situation is far from unlikely in real systems and may be due to several causes, e.g., i) shared pieces of equipment (e.g., components in different systems are fed from the same electrical bus) or ii) physical interactions (e.g., failures of some component create extreme environmental stresses, which increase the probability-chance of multiple-component failures). Instead, in Configuration T3 unknown objective dependence is assumed between $\mathrm{BE} B_{4}$ (i.e., an event representing the failure of a protective or automation system) and $\mathrm{BE} B_{5}$ (i.e., an event dominated by a human error): in real systems, this situation may occur, e.g., when an operator turns off a protection system (event $B_{4}$ ) after failing to correctly diagnose the conditions of a plant (event $B_{5}$ ).

Finally, in Analysis 2 only 'extreme' situations are considered: in particular, in Configurations $\mathrm{T} 1, \mathrm{~T} 2$ and $\mathrm{T} 3$ states of independence, total (perfect) dependence and unknown epistemic dependence, respectively, are assumed between all the probabilities (chances) of all the BEs of the FT.

\begin{tabular}{|c|c|c|c|c|}
\hline \multicolumn{5}{|c|}{ Analysis 1 - Unknown (ukn) epistemic dependence between the probabilities (chances) of the BEs } \\
\hline & Configuration & Events and corresp & nding states of objective $(o b j)$ dependence & Cases \\
\hline \multirow{5}{*}{$\begin{array}{l}\text { Simple configurations: } \\
\text { pairs of Basic Events } \\
\text { (BEs) }\end{array}$} & $\mathrm{C} 1$ & $Z=\left(B_{1} \cap{ }_{o b j} B_{6}\right)^{u k n}$ & \multirow{5}{*}{$o b j=i n d, p e r f, o p p, u k n($ see Section 2.1) } & \multirow{5}{*}{ A, B } \\
\hline & $\mathrm{C} 2$ & $Z=\left(B_{1} \cap \cap_{o b j} B_{5}\right)^{u k n}$ & & \\
\hline & $\mathrm{C} 3$ & $Z=\left(B_{2} \cap{ }_{o b j} B_{5}\right)^{u k n}$ & & \\
\hline & $\mathrm{C} 4$ & $Z=\left(B_{4} \cup \cup_{o b j} B_{5}\right)^{l k n}$ & & \\
\hline & $\mathrm{C} 5$ & $Z=\left(B_{2} \cup \cup_{o b j} B_{3}\right)^{u k n}$ & & \\
\hline \multirow{4}{*}{ Top Event (TE) $X$} & T1 & $X=\left[\left(B_{1} \cup \cup_{\text {ind }} B_{2} \cup\right.\right.$ & ind $\left.\left.B_{3}\right) \cap_{\text {ind }}\left(B_{4} \cup_{\text {ind }} B_{5} \cup_{\text {ind }} B_{6}\right)\right]^{u k n}$ & \multirow{4}{*}{ B } \\
\hline & $\mathrm{T} 2$ & Positive (pos) object & ve dependence between $B_{1}$ and $B_{6}$ & \\
\hline & T3 & $X=\left[\left(B_{1} \cup \cup_{\text {ind }} B_{2} \cup\right.\right.$ & ind $\left.\left.B_{3}\right) \cap_{\text {ind }}\left(B_{4} \cup_{u k n} B_{5} \cup_{\text {ind }} B_{6}\right)\right]^{u k n}$ & \\
\hline & T4 & $X=\left[\left(B_{1} \cup{ }_{u k n} B_{2} \cup\right.\right.$ & $\left.\left.u k n B_{3}\right) \cap_{u k n}\left(B_{4} \cup_{u k n} B_{5} \cup_{u k n} B_{6}\right)\right]^{u k n}$ & \\
\hline \multicolumn{5}{|c|}{ Analysis 2 - Objective independence (ind) between the BEs } \\
\hline & Configuration & Events and corresp & onding states of epistemic $($ epi $)$ dependence & Cases \\
\hline \multirow{5}{*}{$\begin{array}{l}\text { Simple configurations: } \\
\text { pairs of Basic Events } \\
\text { (BEs) }\end{array}$} & $\mathrm{C} 1$ & $Z=\left(B_{1} \cap_{\text {ind }} B_{6}\right)^{e p i}$ & \multirow{5}{*}{ epi $=$ ind, perf,$u k n($ see Section 2.2) } & \multirow{5}{*}{$\mathrm{A}, \mathrm{B}$} \\
\hline & $\mathrm{C} 2$ & $Z=\left(B_{1} \cap_{\text {ind }} B_{5}\right)^{e p i}$ & & \\
\hline & $\mathrm{C} 3$ & $Z=\left(B_{2} \cap_{\text {ind }} B_{5}\right)^{e p i}$ & & \\
\hline & $\mathrm{C} 4$ & $Z=\left(B_{4} \cup \text { ind } B_{5}\right)^{e p i}$ & & \\
\hline & $\mathrm{C} 5$ & $Z=\left(B_{2} \cup \cup_{\text {ind }} B_{3}\right)^{e p i}$ & & \\
\hline \multirow{2}{*}{ Top Event (TE) $X$} & $\mathrm{~T} 1$ & $X=\left[\left(B_{1} \cup_{\text {ind }} B_{2} \cup\right.\right.$ & ind $\left.\left.B_{3}\right) \cap_{\text {ind }}\left(B_{4} \cup_{\text {ind }} B_{5} \cup_{\text {ind }} B_{6}\right)\right]^{\text {ind }}$ & \multirow{2}{*}{$B$} \\
\hline & $\mathrm{T} 2$ & $X=\left[\left(B_{1} \cup \cup_{\text {ind }} B_{2} \cup\right.\right.$ & ind $\left.\left.B_{3}\right) \cap_{\text {ind }}\left(B_{4} \cup_{\text {ind }} B_{5} \cup_{\text {ind }} B_{6}\right)\right]^{p e}$ & \\
\hline
\end{tabular}




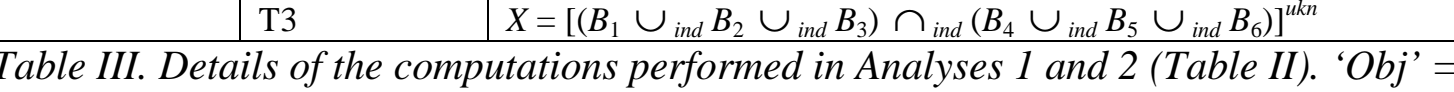

'objective'; 'epi' = 'epistemic'; 'ind' = 'independence'; 'perf' = 'perfect'; 'opp' = 'opposite'; 'ukn' = 'unknown'

\subsection{Quantitative indicators}

Two quantitative indicators are here introduced to evaluate the effects that different states of (objective and state-of-knowledge) dependence between the BEs (Section 3.2) have on the probability (chance) $P(Z)$ of an event $Z$ of interest (e.g., in our case the TE $X$ ): i) the interval $\left[\underline{p}_{Z}^{0.95}, \bar{p}_{Z}^{0.95}\right]$ for the 95 -th percentile $P(Z)^{0.95}$ of $P(Z)$, and ii) the relative average distance $d_{Z}$ between the upper and lower Cumulative Distribution Functions (CDFs) $\bar{F}^{P(z)}$ and $\underline{F}^{P(z)}$.

The interval $\left[\underline{p}_{Z}^{0.95}, \bar{p}_{Z}^{0.95}\right]$ for the 95-th percentile $P(Z)^{0.95}$ of $P(Z)$ is defined as

$\left[\underline{p}_{Z}^{0.95}, \bar{p}_{Z}^{0.95}\right]=\left[\left(\bar{F}^{P(Z)}\right)^{-1}(0.95),\left(\underline{F}^{P(Z)}\right)^{-1}(0.95)\right]$,

where $\left[\bar{F}^{P(z)}\right]^{-1}$ and $\left[\underline{F}^{P(z)}\right]^{-1}$ are the inverse functions of the upper and lower CDFs $\bar{F}^{P(z)}$ and $\underline{F}^{P(Z)}$, respectively, of $P(Z)$. It is worth noting that in a risk analysis context, $\bar{p}_{Z}^{0.95}=$ $\left(F^{P(z)}\right)^{-1}(0.95)$ is the interesting quantity since it guarantees that the probability $P\left[P(Z) \leq \bar{p}_{Z}^{0.95}\right]$ that the true value of $P(Z)$ is lower than $\bar{p}_{Z}^{0.95}=\left(\underline{F}^{P(Z)}\right)^{-1}(0.95)$ is greater than or equal to 0.95 . Thus, $\bar{p}_{Z}^{0.95}=\left(\underline{F}^{P(Z)}\right)^{-1}(0.95)$ can be interpreted as a conservative assignment of the 95-th percentile $P(Z)^{0.95}$ (i.e., a conservative estimate of risk) with respect to the imprecision arising from the input BEs of the FT: obviously, the larger the value of $\bar{p}_{Z}^{0.95}$, the larger the risk associated to the system.

The relative average distance $d_{Z}$ between the upper and lower CDFs $\bar{F}^{P(Z)}$ and $\underline{F}^{P(Z)}$ of $P(Z)$ is defined as 
$d_{Z}=\frac{\int_{0}^{1} d_{Z}(\beta) d \beta}{E\left[P(Z)^{I N S}\right]}=\frac{\int_{0}^{1}\left[\left(\underline{F}^{P(Z)}\right)^{-1}(\beta)-\left(\bar{F}^{P(Z)}\right)^{-1}(\beta)\right] d \beta}{E\left[P(Z)^{I N S}\right]}$,

where $\left[\bar{F}^{P(Z)}\right]^{-1}$ and $\left[\underline{F}^{P(Z)}\right]^{-1}$ are defined above; $d_{Z}(\beta)=\left(\underline{F}^{P(Z)}\right)^{-1}(\beta)-\left(\bar{F}^{P(Z)}\right)^{-1}(\beta)$ is the width of the interval $\left[\underline{p}_{Z}^{\beta}, \bar{p}_{Z}^{\beta}\right]$ for the $\beta$-th percentile $P(Z)^{\beta}$ of $P(Z)$ (in other words, $d_{Z}(\beta)$ is the distance between the upper and lower CDFs $\bar{F}^{P(Z)}$ and $\underline{F}^{P(Z)}$ of $P(Z)$ computed at cumulative probability level $\beta$ along the real 'horizontal' axis; it is straightforward to notice that $d_{Z}(\beta)$ can take values between 0 and 1 because it is the distance between the upper and lower values of the $\beta$-th percentile of $P(Z)$, which obviously takes values between 0 and 1 ); finally, $E\left[P(Z)^{I N S}\right]$ is the expected value of the probability distribution $f^{P(Z)^{I N S}}\left(p_{Z}^{I N S}\right)$ obtained by transforming the upper and lower CDFs $\bar{F}^{P(Z)}$ and $\underline{F}^{P(Z)}$ of $P(Z)$ according to the principle of insufficient reason ${ }^{(85)}$. The sampling procedure for estimating $E\left[P(Z)^{I N S}\right]$ is:

i. transform the upper and lower $\mathrm{CDFs} \bar{F}^{P(Z)}$ and $\underline{F}^{P(Z)}$ of $P(Z)$ into the (unique) probability distribution $f^{P(Z)^{I N S}}\left(p_{Z}^{I N S}\right)^{(85,86)}$ :

a. sample $N_{I N S}$ random realizations $\left\{u_{k}: k=1,2, \ldots, N_{I N S}\right\}$ from a uniform probability distribution on $[0,1)$ and consider the corresponding intervals $\left[\left(\bar{F}^{P(Z)}\right)^{-1}\left(u_{k}\right),\left(\underline{F}^{P(Z)}\right)^{-1}\left(u_{k}\right)\right], k=1,2, \ldots, N_{I N S}$

b. sample a random realization $p_{Z, k}^{I N S}$ for $P(Z)^{I N S}$ from a uniform probability distribution on each interval $\left[\left(\bar{F}^{P(Z)}\right)^{-1}\left(u_{k}\right),\left(\underline{F}^{P(Z)}\right)^{-1}\left(u_{k}\right)\right], k=1,2, \ldots, N_{I N S}$ : the distribution resulting from the collection of the realizations $p_{Z, k}^{I N S}, k=1,2, \ldots$, $N_{I N S}$, is an empirical estimate for $\left.f^{P(Z)^{I N S}}\left(p_{Z}^{I N S}\right)\right)$;

ii. estimate $E\left[P(Z)^{I N S}\right]$ as $1 / N_{I N S} \cdot \sum_{k=1}^{N_{I N S}} p_{Z, k}^{I N S}$. 
Other methods for transforming the upper and lower CDFs $\bar{F}^{P(Z)}$ and $\underline{F}^{P(Z)}$ of $P(Z)$ into a (unique) probability distribution are available in ${ }^{(36,85,87,88)}$.

It is worth noting that the quantity $d_{Z}(20)$ provides a measure of the average distance (i.e., separation) between the upper and lower CDFs $\bar{F}^{P(Z)}$ and $\underline{F}^{P(Z)}$ of $P(Z)$, computed along the real 'horizontal' axis. In this sense, it is also an indicator of the uncertainty (i.e., imprecision) 'contained' in the distribution of $P(Z)$ : the larger the average distance $d_{Z}(20)$, the larger the uncertainty (imprecision) associated to $P(Z)$.

Finally, notice that the expected value $E\left[P(Z)^{I N S}\right]$ in (20) is simply chosen as a numerical indicator of the approximate "location" of the upper and lower $\mathrm{CDFs} \bar{F}^{P(Z)}$ and $\underline{F}^{P(Z)}$ on the 'horizontal' axis: in other words, it is taken as a numerical indicator of the order of magnitude of $P(Z)$. In this view, $E\left[P(Z)^{I N S}\right]$ serves the main purpose of a normalization factor for the integral $\int_{0}^{1}\left[\left(\underline{F}^{P(Z)}\right)^{-1}(\beta)-\left(\bar{F}^{P(z)}\right)^{-1}(\beta)\right] d \beta$, whose magnitude is obviously dependent on the magnitude of $P(Z)$ and, thus, on the magnitude of the BE probabilities (chances). In this way, such normalization factor allows a fair comparison between values of the distance $d_{Z}(20)$ computed in Cases A and B (Section 3.1), where the BE probabilities (chances) differ by several orders of magnitude.

\section{APPLICATION}

In this Section, the methods described in Section 2 for handling dependences in FTA are applied to the example of Section 3. In particular, Section 4.1 contains the results of Analysis 1 (Table III in Section 3.2), whereas Section 4.2 reports the results of Analysis 2 (Table III in Section 3.2). 


\subsection{Studying the effects of objective (aleatory) dependences between the basic events}

Table IV reports the values of the indicators $\left[\underline{p}_{Z}^{0.95}, \bar{p}_{Z}^{0.95}\right]$ (19) and $d_{Z}(20)$ obtained for the events $Z=\left(B_{1} \bigcap_{o b j} B_{6}\right)^{u k n},\left(B_{1} \cap_{o b j} B_{5}\right)^{u k n},\left(B_{2} \cap_{o b j} B_{5}\right)^{u k n},\left(B_{4} \cup_{o b j} B_{5}\right)^{u k n}$ and $\left(B_{2} \cup_{o b j} B_{3}\right)^{u k n}$ (Configurations C1-C5 of Analysis 1 in Table III) under the assumptions of independence ('obj' = 'ind'), perfect ('obj' = 'perf'), opposite ('obj' = 'opp') and unknown ('obj' = 'ukn') objective dependence, with reference to Cases A and B (Section 3.1); the estimates of $E\left[P(Z)^{I N S}\right]$ are also shown for completeness. In addition, only for illustration purposes Figure 6 depicts the upper and lower Cumulative Distribution Functions (CDFs) $\bar{F}^{P\left[\left(B_{1} \cap_{o b j} B_{5}{ }^{\mu k n}\right]\right.}$, $\bar{F}^{P\left[\left(B_{4} \cup_{o b j} B_{5}\right)^{\mu k n}\right]}, \underline{F}^{P\left[\left(B_{1} \cap_{o b j} B_{5}\right)^{k k n}\right]}$ and $\underline{F}^{P\left[\left(B_{4} \cup_{o b j} B_{5}\right)^{k k n}\right]}$ obtained for events $\left(B_{1} \cap_{o b j} B_{5}\right)^{u k n}$ (top) and $\left(B_{4}\right.$ $\left.\cup_{o b j} B_{5}\right)^{u k n}$ (bottom), respectively, under the assumptions of independence (solid lines), perfect (dashed lines), opposite (dotted lines) and unknown (dot-dashed lines) objective dependence, with reference to Cases A (left) and B (right). Notice that by construction $\bar{F}^{P\left[\left(B_{1} \cap_{u k n} B_{5}\right)^{\mu k n}\right]}=\bar{F}^{P\left[\left(B_{1} \cap_{p e r f} B_{5}\right)^{\mu k n}\right]}$ and $\underline{F}^{P\left[\left(B_{1} \cap_{u k n} B_{5}\right)^{u k n}\right]}=\underline{F}^{P\left[\left(B_{1} \cap_{\text {opp }} B_{5}\right)^{k k n}\right]}$, whereas $\bar{F}^{P\left[\left(B_{4} \cup_{u k n} B_{5}\right)^{u k n}\right]}=$ $\bar{F}^{P\left[\left(B_{4} \cup_{\text {opp }} B_{5}\right)^{\mu k n}\right]}$ and $\underline{F}^{P\left[\left(B_{4} \cup_{u k n} B_{5}\right)^{\mu k n}\right]}=\underline{F}^{P\left[\left(B_{4} \cup_{\text {perf }} B_{5}\right)^{k k n}\right]}$ (see (7) and (8)): however, only for clarity of illustration the corresponding lines in Figure 6 are not overlapped.

We start by analyzing those cases where the BEs are linked by AND-gates, i.e., $Z=\left(B_{1} \cap_{o b j}\right.$ $\left.B_{6}\right)^{u k n},\left(B_{1} \cap_{o b j} B_{5}\right)^{u k n},\left(B_{2} \bigcap_{o b j} B_{5}\right)^{u k n}$ (Configurations C1-C3 in Table III). It can be seen that in Case A the upper bounds $\bar{p}_{\left(B_{1} \cap_{o b j} B_{6}\right)^{\mu k n}}^{0.95}, \bar{p}_{\left(B_{1} \cap_{o b j} B_{5}\right)^{k k n}}^{0.95}$ and $\bar{p}_{\left(B_{2} \cap_{o b j} B_{5}\right)^{k k n}}^{0.95}$ of the 95-th percentiles $P\left[\left(B_{1} \cap \cap_{o b j} B_{6}\right)^{u k n}\right]^{0.95}, P\left[\left(B_{1} \cap_{o b j} B_{5}\right)^{u k n}\right]^{0.95}$ and $P\left[\left(B_{2} \cap_{o b j} B_{5}\right)^{u k n}\right]^{0.95}$ are $0.1528,0.1557$ and 0.1760 , respectively, under the assumption of independence, whereas they are $0.3461,0.3462$ and 0.3941 , respectively, under the assumption of unknown dependence. Thus, the 
assumption of independence would lead to underestimating the upper bounds of the 95-th quantiles (and, thus, the risk associated to the system) by 2.27, 2.22 and 2.24 times, respectively. These considerations are reflected also by the analysis of the relative average distances $d_{\left(B_{1} \cap_{o b j} B_{6}\right)^{k k n}}, d_{\left(B_{1} \cap_{o b j} B_{5}\right)^{k k n}}$ and $d_{\left(B_{2} \cap_{o b j} B_{5}\right)^{k k n}}$ between the upper and lower CDFs $\bar{F}^{P\left[\left(B_{1} \cap_{o b j} B_{6}\right)^{k k n}\right]}, \bar{F}^{P\left[\left(B_{1} \cap_{o b j} B_{5}\right)^{k k n}\right]}, \bar{F}^{P\left[\left(B_{2} \cap_{o b j} B_{5}\right)^{k k n}\right]}, \underline{F}^{P\left[\left(B_{1} \cap_{o b j} B_{6}\right)^{k k n}\right]}, \underline{F}^{P\left[\left(B_{1} \cap_{o b j} B_{5}\right)^{k k n}\right]}$ and $\underline{F}^{P\left[\left(B_{2} \cap_{o b j} B_{5}\right)^{k k n}\right]}$, respectively. Actually, as before the assumption of independence leads to underestimating the uncertainty (imprecision) 'contained' in the distributions of the probabilities $P\left[\left(B_{1} \cap_{o b j} B_{6}\right)^{u k n}\right], \quad P\left[\left(B_{1} \cap_{o b j} B_{5}\right)^{u k n}\right]$ and $P\left[\left(B_{2} \cap_{o b j} B_{5}\right)^{u k n}\right]$ by 4.25, 2.61 and 2.65 times, respectively.

This underestimation is much more significant in Case B. Actually, the values of $\bar{p}_{\left(B_{1} \cap_{o b j} B_{6}\right)^{\mu k n}}^{0.95}$, $\bar{p}_{\left(B_{1} \cap_{o b j} B_{5}\right)^{\mu k n}}^{0.95}$ and $\bar{p}_{\left(B_{2} \cap_{o b j} B_{5}\right)^{\mu k n}}^{0.95}$ are $9.2078 \cdot 10^{-5}, 6.9995 \cdot 10^{-5}$ and $7.0080 \cdot 10^{-5}$, respectively, under the assumption of independence, whereas they are $8.1876 \cdot 10^{-3}, 8.8002 \cdot 10^{-3}$ and $7.8810 \cdot 10^{-3}$, respectively, under the assumption of unknown dependence. Thus, the assumption of independence leads to underestimating the upper bounds of the 95-th quantiles (and, thus, the risk associated to the system) by $89.02,125.70$ and 112.60 times, respectively. Again, these considerations are reflected by the analysis of the relative average distances $d_{\left(B_{1} \cap_{o b j} B_{6}\right)^{k k n}}$, $d_{\left(B_{1} \cap_{o b j} B_{5}\right)^{k k n}}$ and $d_{\left(B_{2} \cap_{o b j} B_{5}\right)^{\mu k n}}$. Actually, as before the assumption of independence leads to underestimating the uncertainty (imprecision) associated to the distributions of $P\left[\left(B_{1} \cap_{o b j} B_{6}\right)^{u k n}\right], \quad P\left[\left(B_{1} \cap_{o b j} B_{5}\right)^{u k n}\right]$ and $P\left[\left(B_{2} \cap_{o b j} B_{5}\right)^{u k n}\right]$ by $136.89,164.80$ and 140.99 times, respectively. A visual representation of these results is given in Figure 6, top: actually, it can be seen that the upper and lower CDFs $\bar{F}^{P\left[\left(B_{1} \cap_{u k n} B_{5}\right)^{k k n}\right]}$ and $\underline{F}^{P\left[\left(B_{1} \cap_{u k n} B_{5}\right)^{u k n}\right]}$ of 
$P\left[\left(B_{1} \cap_{u k n} B_{5}\right)^{u k n}\right]$ (dashed lines) completely envelop the upper and lower CDFs $\left.\bar{F}^{P\left[\left(B_{1} \cap_{i n d} B_{5}\right)^{u k n}\right.}\right]$ and $\underline{F}^{P\left[\left(B_{1} \cap_{\text {ind }} B_{5}\right)^{\mu k n}\right]}$ of $P\left[\left(B_{1} \cap_{\text {ind }} B_{5}\right)^{u k n}\right]$ (solid lines) in both Cases A (left) and B (right).

The facts that i) the assumption of objective independence leads to a consistent underestimation of risk and ii) such underestimation is more dramatic in Case B than in Case A are explained as follows. The probability (chance) of the conjunction of two independent events, say $B_{1}$ and $B_{5}$, is given by the product of the corresponding probabilities (chances) $P\left(B_{1}\right)$ and $P\left(B_{5}\right)$, i.e., $P\left(B_{1} \cap_{\text {ind }} B_{5}\right)=P\left(B_{1}\right) \cdot P\left(B_{5}\right)$ (see (1)): thus, if $P\left(B_{1}\right)$ and $P\left(B_{5}\right)$ are of the order of $10^{-n}$, then $P\left(B_{1} \cap_{\text {ind }} B_{5}\right)$ is of the order of $10^{-2 n}$. Instead, if no assumption at all about the state of objective dependence between $B_{1}$ and $B_{5}$ can be made, only (extreme and best possible) lower and upper bounds on $P\left(B_{1} \cap B_{5}\right)$ can be computed as $P\left(B_{1} \cap_{u k n} B_{5}\right)=$ $\left[\underline{P}\left(B_{1} \cap_{u k n} B_{5}\right), \bar{P}\left(B_{1} \cap_{u k n} B_{5}\right)\right] \quad=\quad\left[\underline{P}\left(B_{1} \cap_{\text {opp }} B_{5}\right), \bar{P}\left(B_{1} \cap_{\text {perf }} B_{5}\right)\right]=$ $\left[\max \left\{P\left(B_{1}\right)+P\left(B_{5}\right)-1,0\right\}, \min \left\{P\left(B_{1}\right), P\left(B_{5}\right)\right\}\right]$ (see (7)). In this case, if $P\left(B_{1}\right)$ and $P\left(B_{5}\right)$ are of the order of $10^{-n}$, then the upper bound $\bar{P}\left(B_{1} \cap_{u k n} B_{5}\right)=\min \left\{P\left(B_{1}\right), P\left(B_{5}\right)\right\}$ (which represents the most conservative estimate of risk) is still of the order of $10^{-n}$. As a consequence, $\bar{P}\left(B_{1} \cap_{u k n} B_{5}\right) \approx 10^{-n}$ is approximately $n$ orders of magnitude larger than $P\left(B_{1} \cap_{\text {ind }} B_{5}\right) \approx 10^{-2 n}$, which explains also why the difference between $P\left(B_{1} \cap_{\text {ind }} B_{5}\right)$ and $\bar{P}\left(B_{1} \cap_{u k n} B_{5}\right)$ dramatically increases as $P\left(B_{1}\right)$ and $P\left(B_{5}\right)$ decrease (i.e., as $n$ increases).

Different situations arise in the cases where the BEs are linked by OR-gates, i.e., $Z=\left(B_{4} \cup_{o b j}\right.$ $\left.B_{5}\right)^{u k n}$ and $\left(B_{2} \cup_{o b j} B_{3}\right)^{u k n}$ (Configurations C4 and C5 in Table III). It can be seen that in Case A the values of $\bar{p}_{\left(B_{4} \cup_{o b j} B_{5}\right)^{k a n}}^{0.95}$ and $\bar{p}_{\left(B_{2} \cup_{o b j} B_{3}\right)^{k n}}^{0.05}$ are 0.6670 and 0.6970 , respectively, under the assumption of independence, whereas they are 0.8401 and 0.8941 , respectively, under the assumption of unknown dependence. Thus, the assumption of independence leads to 
underestimating the upper bounds of the 95-th quantiles (and, thus, the risk associated to the system) by about 1.26 and 1.28 times, respectively. These considerations are reflected also by the values of the relative average distances $d_{\left(B_{4} \cup_{o b j} B_{5}\right)^{\mu k n}}$ and $d_{\left(B_{2} \cup_{o b j} B_{3}\right)^{\mu k n}}$ between the upper and lower CDFs $\bar{F}^{P\left[\left(B_{4} \cup_{o b j} B_{5}\right)^{\mu k n}\right]}, \quad \bar{F}^{P\left[\left(B_{2} \cup_{o b j} B_{3}\right)^{\mu k n}\right]}, \underline{F}^{P\left[\left(B_{4} \cup_{o b j} B_{5}\right)^{\mu k n}\right]}$ and $\underline{F}^{P\left[\left(B_{2} \cup_{o b j} B_{3}\right)^{k k n}\right]}$, respectively. Actually, as before, the assumption of independence leads to underestimating the uncertainty (imprecision) 'contained' in the distributions of $P\left[\left(B_{4} \cup_{o b j} B_{5}\right)^{u k n}\right]$ and $P\left[\left(B_{2} \cup_{o b j} B_{3}\right)^{u k n}\right]$ by 1.45 and 1.85 times. Notice that the magnitude of such underestimations is not negligible, but it is much less relevant than for the cases where BEs are linked by AND-gates.

In Case $\mathrm{B}$, the values of $\bar{p}_{\left(B_{4} \cup_{o b j} B_{5}\right)^{k k n}}^{0.95}$ and $\bar{p}_{\left(B_{2} \cup_{o b j} B_{3}\right)^{k k n}}^{0.95}$ are $1.6685 \cdot 10^{-2}$ and $1.7772 \cdot 10^{-2}$, respectively, under the assumption of independence, whereas they are $1.6763 \cdot 10^{-2}$ and $1.7881 \cdot 10^{-2}$, respectively, under the assumption of unknown dependence. Thus, in this case the assumption of independence leads to a very slight underestimation of the upper bounds of the 95-th quantiles (and, thus, of the risk associated to the system), i.e., only by about 1.01 and 1.02 times, respectively. Instead, the values of the relative average distances $d_{\left(B_{4} \cup \cup_{o j j} B_{5}\right)^{a k n}}$ and $d_{\left(B_{2} \cup \text { obj } B_{3}\right)^{\mu k n}}$ are 1.0216 and 0.6381 , respectively, under the assumption of independence, whereas they are 1.1676 and 0.8076 , respectively, under the assumption of unknown dependence: in other words, the uncertainty (imprecision) associated to distributions of $P\left[\left(B_{4} \cup_{o b j} B_{5}\right)^{u k n}\right]$ and $P\left[\left(B_{2} \cup_{o b j} B_{3}\right)^{u k n}\right]$ is underestimated by about 1.14 and 1.27 times. Thus, although the risk estimates are comparable, the underestimation of the uncertainty (imprecision) associated to the distributions of $P\left[\left(B_{4} \cup_{o b j} B_{5}\right)^{u k n}\right]$ and $P\left[\left(B_{2} \cup_{o b j} B_{3}\right)^{u k n}\right]$ is not negligible. A visual representation of these results is given in Figure 6, bottom right. Actually, it can be seen that the lower CDFs $\underline{F}^{P\left[\left(B_{4} \cup_{u k n} B_{5}\right)^{u k n}\right]}$ (dashed line) and $\underline{F}^{P\left[\left(B_{4} \cup_{\text {ind }} B_{5}\right)^{u k n}\right]}$ (solid line) (i.e., the CDFs used to estimate the upper bounds of the 95-th quantiles of $P\left[\left(B_{4} \cup_{u k n} B_{5}\right)^{u k n}\right]$ 
and $P\left[\left(B_{4} \cup_{\text {ind }} B_{5}\right)^{u k n}\right]$, respectively) almost coincide; on the contrary, the upper CDF $\left.\left.\bar{F}^{P\left[\left(B_{4} \cup u k n\right.\right.} B_{5}\right)^{u k n}\right]$ (dashed line) lies consistently above the upper CDF $\bar{F}^{P\left[\left(B_{4} \cup \text { ind } B_{5}\right)^{u k n}\right]}$ (solid line).

These results are explained as follows. The probability (chance) of the disjunction of two independent events, say $B_{4}$ and $B_{5}$, is given by $P\left(B_{4} \cup_{\text {ind }} B_{5}\right)=P\left(B_{4}\right)+P\left(B_{5}\right)-$ $P\left(B_{4}\right) \cdot P\left(B_{5}\right)$ (see (2)). Instead, if no assumptions at all about the state of objective dependence between $B_{4}$ and $B_{5}$ can be made, only (extreme and best possible) lower and upper bounds on $P\left(B_{4} \cup B_{5}\right)$ can be computed as $P\left(B_{4} \cup \cup_{u k n} B_{5}\right)=$ $\left[\underline{P}\left(B_{4} \cup_{u k n} B_{5}\right), \bar{P}\left(B_{4} \cup_{u k n} B_{5}\right)\right] \quad=\quad\left[\underline{P}\left(B_{4} \cup \cup_{p e r f} B_{5}\right), \bar{P}\left(B_{4} \cup_{\text {opp }} B_{5}\right)\right]=$ $\left[\max \left\{P\left(B_{4}\right), P\left(B_{5}\right)\right\}, \min \left\{1, P\left(B_{4}\right)+P\left(B_{5}\right)\right\}\right]$ (see (8)). If both $P\left(B_{4}\right)$ and $P\left(B_{5}\right)$ are of the order of $10^{-n}$ (with $n>1$, like in the present Case B), then $P\left(B_{4} \cup_{\text {ind }} B_{5}\right)=P\left(B_{4}\right)+P\left(B_{5}\right)-$ $P\left(B_{4}\right) \cdot P\left(B_{5}\right) \approx P\left(B_{4}\right)+P\left(B_{5}\right)=2 \cdot 10^{-n}$. In addition, it is evident that $P\left(B_{4} \cup_{u k n} B_{5}\right)=$ $\left[\max \left\{P\left(B_{4}\right), P\left(B_{5}\right)\right\}, \min \left\{1, P\left(B_{4}\right)+P\left(B_{5}\right)\right\}\right] \approx\left[10^{-n}, 2 \cdot 10^{-n}\right]$. This means that if both $P\left(B_{4}\right)$ and $P\left(B_{5}\right)$ are quite small (i.e., if $\left.n>>1\right)$, then the value of $P\left(B_{4} \cup_{\text {ind }} B_{5}\right)$ is comparable to that of $\bar{P}\left(B_{4} \cup_{u k n} B_{5}\right)$, i.e., $P\left(B_{4} \cup_{\text {ind }} B_{5}\right) \approx \bar{P}\left(B_{4} \cup_{u k n} B_{5}\right) \approx 2 \cdot 10^{-n}$ : in other words, two radically different assumptions about the state of objective dependence between $B_{4}$ and $B_{5}$ provide a comparable risk estimate. On the contrary, the uncertainty (imprecision) 'contained' in the distributions of $P\left(B_{4} \cup_{\text {ind }} B_{5}\right)$ and $P\left(B_{4} \cup_{u k n} B_{5}\right)$ is obviously quite different: actually, the interval $P\left(B_{4} \cup_{u k n} B_{5}\right) \approx\left[10^{-n}, 2 \cdot 10^{-n}\right]$ 'completely envelops' the estimate $P\left(B_{4} \cup_{\text {ind }} B_{5}\right) \approx$ $2 \cdot 10^{-n}$

\begin{tabular}{|c|c|c|c|c|c|}
\hline \multicolumn{6}{|c|}{ Analysis 1 - Unknown (ukn) epistemic dependence between the probabilities (chances) of the BEs } \\
\hline \multicolumn{6}{|c|}{ Case A } \\
\hline & & \multicolumn{4}{|c|}{ State of objective (obj) dependence } \\
\hline Event $Z$ & Indicators & Independence (ind) & Perfect (perf) & Opposite (opp) & Unknown (ukn) \\
\hline \multirow{3}{*}{$\begin{array}{c}\left(B_{1} \cap_{o b j} B_{6}\right)^{u k n} \\
(\mathbf{C} 1)\end{array}$} & $E\left[P(Z)^{I N S}\right]$ & 0.0583 & 0.1954 & 0 & 0.1069 \\
\hline & $d_{z}$ & 0.8634 & 0.6321 & I & 3.6672 \\
\hline & 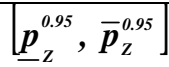 & {$[0.0492,0.1528]$} & {$[0.2205,0.3461]$} & 0 & {$[0,0.3461]$} \\
\hline
\end{tabular}




\begin{tabular}{|c|c|c|c|c|c|}
\hline \multirow{3}{*}{$\begin{array}{c}\left(\boldsymbol{B}_{1} \cap_{o b j} \boldsymbol{B}_{5}\right)^{u k n} \\
(\mathbf{C} 2)\end{array}$} & $E\left[P(Z)^{I N S}\right]$ & 0.0553 & 0.1627 & 0 & 0.1068 \\
\hline & $d_{z}$ & 1.4779 & 1.8426 & 1 & 3.8630 \\
\hline & $\underline{\boldsymbol{p}}_{z}^{0.95}, \overline{\boldsymbol{p}}_{z}^{0.95}$ & {$[0.0243,0.1557]$} & {$[0.1564,0.3462]$} & 0 & {$[0,0.3462]$} \\
\hline \multirow{3}{*}{$\begin{array}{c}\left(\boldsymbol{B}_{2} \cap_{o b j} \boldsymbol{B}_{5}\right)^{u k n} \\
(\mathbf{C} \mathbf{3})\end{array}$} & $\boldsymbol{E}\left[\boldsymbol{P}(\boldsymbol{Z})^{I N S}\right]$ & 0.0715 & 0.2068 & 0 & 0.1573 \\
\hline & $d_{z}$ & 1.6629 & 3.0140 & 1 & 4.3991 \\
\hline & 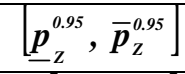 & {$[0.0188,0.1760]$} & {$[0.1220,0.3941]$} & 0 & {$[0,0.3941]$} \\
\hline \multirow{3}{*}{$\left(\boldsymbol{B}_{4} \cup_{\text {obj }} \boldsymbol{B}_{5}\right)^{u k n}$} & $E\left[\boldsymbol{P}(\boldsymbol{Z})^{I N S}\right]$ & 0.3933 & 0.2645 & 0.4538 & 0.4219 \\
\hline & $d_{z}$ & 0.9325 & 0.5493 & 1.1871 & 1.3495 \\
\hline & $\underline{\boldsymbol{p}}_{Z}^{0.95}, \overline{\boldsymbol{p}}_{Z}^{0.95}$ & {$[0.3100,0.6670]$} & {$[0.2500,0.4401]$} & {$[0.3302,0.8401]$} & {$[0.2500,0.8401]$} \\
\hline \multirow{3}{*}{$\begin{array}{c}\left(\boldsymbol{B}_{2} \cup_{o b j} \boldsymbol{B}_{3}\right)^{u k n} \\
(\mathbf{C} 5)\end{array}$} & $\boldsymbol{E}\left[\boldsymbol{P}(\boldsymbol{Z})^{I N S}\right]$ & 0.5272 & 0.3873 & 0.6251 & 0.5750 \\
\hline & $d_{z}$ & 0.5161 & 0.2419 & 0.7644 & 0.9542 \\
\hline & {$\left[\underline{p}_{z}^{0.95}, \bar{p}_{z}^{0.95}\right.$} & {$[0.4519,0.6970]$} & {$[0.3910,0.4961]$} & {$[0.4911,0.8941]$} & {$[0.3910,0.8941]$} \\
\hline \multicolumn{6}{|c|}{ Case B } \\
\hline & & \multicolumn{4}{|c|}{ State of objective $(o b j)$ dependence } \\
\hline Event $Z$ & Indicators & Independence (ind) & Perfect (perf) & Opposite (opp) & Unknown (ukn) \\
\hline \multirow{3}{*}{$\begin{array}{c}\left(\boldsymbol{B}_{1} \cap_{o b j} \boldsymbol{B}_{6}\right)^{u k n} \\
(\mathbf{C} 1)\end{array}$} & $\boldsymbol{E}\left[\boldsymbol{P}(\boldsymbol{Z})^{I N S}\right]$ & $2.01 \cdot 10^{-5}$ & $3.00 \cdot 10^{-3}$ & 0 & $1.84 \cdot 10^{-3}$ \\
\hline & $d_{z}$ & 1.3339 & 67.0056 & 1 & 182.6408 \\
\hline & 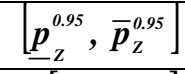 & {$\left[1.41 \cdot 10^{-5}, 9.21 \cdot 10^{-5}\right]$} & {$\left[3.43 \cdot 10^{-3}, 8.19 \cdot 10^{-3}\right]$} & 0 & {$\left[0,8.19 \cdot 10^{-3}\right]$} \\
\hline \multirow{3}{*}{$\begin{array}{c}\left(\boldsymbol{B}_{1} \cap_{o b j} \boldsymbol{B}_{5}\right)^{u k n} \\
(\mathbf{C 2})\end{array}$} & $\boldsymbol{E}\left[\boldsymbol{P}(\boldsymbol{Z})^{I N S}\right]$ & $1.84 \cdot 10^{-5}$ & $2.71 \cdot 10^{-3}$ & 0 & $1.83 \cdot 10^{-3}$ \\
\hline & $d_{z}$ & 1.2028 & 101.9268 & 1 & 198.2254 \\
\hline & {$\left[\underline{p}_{z}^{0.95}, \bar{p}_{z}^{0.95}\right]$} & {$\left[8.75 \cdot 10^{-6}, 7.85 \cdot 10^{-5}\right]$} & {$\left[2.56 \cdot 10^{-3}, 8.80 \cdot 10^{-3}\right]$} & 0 & {$\left[0,8.80 \cdot 10^{-3}\right]$} \\
\hline \multirow{3}{*}{$\begin{array}{c}\left(\boldsymbol{B}_{2} \cap_{o b j} \boldsymbol{B}_{5}\right)^{u k n} \\
(\mathbf{C} 3)\end{array}$} & $\boldsymbol{E}\left[\boldsymbol{P}(\boldsymbol{Z})^{I N S}\right]$ & $2.62 \cdot 10^{-5}$ & $4.14 \cdot 10^{-3}$ & 0 & $3.15 \cdot 10^{-3}$ \\
\hline & $d_{z}$ & 1.7034 & 164.6245 & 1 & 240.1765 \\
\hline & {$\left[\underline{p}_{z}^{0.95}, \bar{p}_{z}^{0.95}\right.$} & {$\left[5.68 \cdot 10^{-6}, 7.01 \cdot 10^{-5}\right]$} & {$\left[2.44 \cdot 10^{-3}, 7.88 \cdot 10^{-3}\right]$} & 0 & {$\left[0,7.88 \cdot 10^{-3}\right]$} \\
\hline \multirow{3}{*}{$\begin{array}{c}\left(\boldsymbol{B}_{4} \cup_{o b j} \boldsymbol{B}_{5}\right)^{u k n} \\
(\mathbf{C 4})\end{array}$} & $E\left[P(Z)^{I N S}\right]$ & $8.93 \cdot 10^{-3}$ & $5.19 \cdot 10^{-3}$ & $9.00 \cdot 10^{-3}$ & $8.33 \cdot 10^{-3}$ \\
\hline & $d_{z}$ & 1.0216 & 0.4700 & 1.0325 & 1.1676 \\
\hline & $\underline{\boldsymbol{p}_{Z}^{0.95}}, \overline{\boldsymbol{p}}_{z}^{0.95}$ & {$\left[6.59 \cdot 10^{-3}, 1.67 \cdot 10^{-2}\right]$} & {$\left[5.00 \cdot 10^{-3}, 8.76 \cdot 10^{-3}\right]$} & {$\left[6.40 \cdot 10^{-3}, 1.68 \cdot 10^{-2}\right]$} & {$\left[5.00 \cdot 10^{-3}, 1.68 \cdot 10^{-2}\right]$} \\
\hline \multirow{3}{*}{$\left(B_{2} \cup_{(\text {C5 } 5)} B_{3}\right)^{u k n}$} & $E\left[P(Z)^{I N S}\right]$ & $1.25 \cdot 10^{-2}$ & $7.74 \cdot 10^{-3}$ & $1.25 \cdot 10^{-2}$ & $1.15 \cdot 10^{-2}$ \\
\hline & $d_{z}$ & 0.6381 & 0.2047 & 0.6468 & 0.8076 \\
\hline & {$\left[\underline{\boldsymbol{p}}_{Z}^{0.95}, \overline{\boldsymbol{p}}_{Z}^{0.95}\right.$} & {$\left[9.83 \cdot 10^{-3}, 1.78 \cdot 10^{-2}\right]$} & {$\left[7.82 \cdot 10^{-3}, 9.92 \cdot 10^{-3}\right]$} & {$\left[9.82 \cdot 10^{-3}, 1.79 \cdot 10^{-2}\right]$} & {$\left[7.82 \cdot 10^{-3}, 1.79 \cdot 10^{-2}\right]$} \\
\hline
\end{tabular}

Table IV. Values of the indicators $\left[\underline{p}_{Z}^{0.95}, \bar{p}_{Z}^{0.95}\right](19)$ and $d_{Z}(20)$ obtained for the simple events $Z=\left(B_{1} \cap_{o b j} B_{6}\right)^{u k n},\left(B_{1} \cap_{o b j} B_{5}\right)^{u k n},\left(B_{2} \cap_{o b j} B_{5}\right)^{u k n},\left(B_{4} \cup \cup_{o b j} B_{5}\right)^{u k n}$ and $\left(B_{2} \cup \text { obj } B_{3}\right)^{u k n}$

(Configurations C1-C5 of Analysis 1 in Table III) under the assumptions of independence, perfect, opposite and unknown objective dependence, with reference to Cases $A$ and $B$; the estimates for $E\left[P(Z)^{I N S}\right]$ are also reported for completeness 

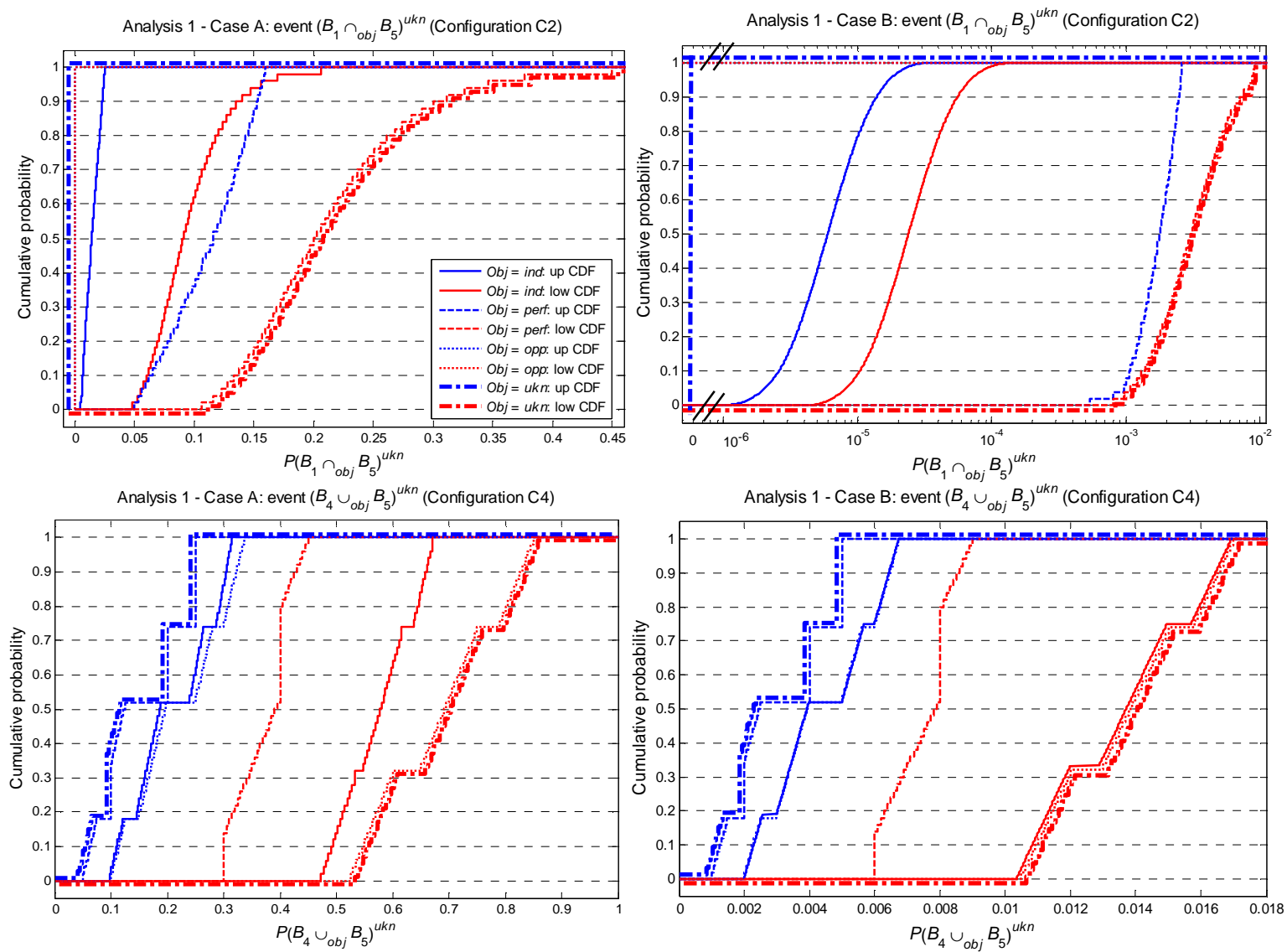

Figure 6. Upper and lower CDFs $\bar{F}^{P\left[\left(B_{1} \cap_{o b j} B_{5}\right)^{k k n}\right]}, \bar{F}^{P\left[\left(B_{4} \cup_{o b j} B_{5}\right)^{\mu k n}\right]}, F^{P\left[\left(B_{1} \cap_{o b j} B_{5}\right)^{k k n}\right]}$ and $\underline{F}^{P\left[\left(B_{4} \cup_{o b j} B_{5}\right)^{k k n}\right]}$ obtained for events $\left(B_{1} \cap_{o b j} B_{5}\right)^{u k n}($ top $)$ and $\left(B_{4} \cup \cup_{o b j} B_{5}\right)^{u k n}$ (bottom), respectively, under the assumptions of independence (solid lines), perfect (dashed lines), opposite (dotted lines) and unknown (dot-dashed lines) objective dependence, with reference to Case A (left) and B (right). Top, right: the value $P\left[\left(B_{1} \cap_{o b j} B_{5}\right)^{u k n}\right]=0$ in Case B is represented out of scale at about $6 \cdot 10^{-7}$ for clarity of illustration

Similar analyses are performed on the probability (chance) $P(X)$ of the TE $X$ of the FT in Figure 4. Table $\mathrm{V}$ reports the values of the indicators $\left[\underline{p}_{X}^{0.95}, \bar{p}_{X}^{0.95}\right]$ (19) and $d_{X}(20)$ obtained for $P(X)$ under different assumptions of objective dependence between the BEs (Configurations T1-T4 in Table III), with reference to Case B; the estimates for $E\left[P(X)^{I N S}\right]$ are also shown for completeness. For illustration purposes, Figure 7 depicts the upper and lower CDFs $\bar{F}^{P(X)}$ and $\underline{F}^{P(X)}$ obtained for $P(X)$ under different assumptions of objective dependence between the BEs (Configurations T1-T4 in Table III). 
These results confirm the considerations drawn by the analysis of the simple Configurations C1-C5 in Table III. For example, it can be seen that the values of the upper bound $\bar{p}_{X}^{0.95}$ on the 95-th quantile $P(X)^{0.95}$ are $7.2275 \cdot 10^{-4}$ and $8.9766 \cdot 10^{-3}$ in Configurations T1 (where all the BEs are considered independent) and $\mathrm{T} 2$ (where BEs $B_{1}$ and $B_{6}$ are considered positively dependent). This means that neglecting an hypothetical state of positive dependence between only one pair of BEs linked by an AND-gate is sufficient for underestimating the upper bound $\bar{p}_{X}^{0.95}$ of the 95-th quantile $P(X)^{0.95}$ (and, thus, the risk associated to the system) by 12.42 times. On the contrary, in Configuration T3 (where no indication at all about the state of objective dependence between BEs $B_{4}$ and $B_{5}$ is available), the value of $\bar{p}_{X}^{0.95}$ is $7.7580 \cdot 10^{-4}$ : thus, in this case even assuming unknown objective dependence between a couple of BEs linked by an OR-gate leads to overestimating the risk associated to the system only by about 1.07 times with respect to the 'baseline' assumption of independence. Finally, Configuration T4 represents the 'extreme' case where unknown objective dependence is assumed between all the BEs of the FT: notice that since in the present Analysis 1 unknown epistemic dependence is also assumed between the probabilities (chances) of all the BEs, Configuration T4 provides the most 'uncertain' and, thus, conservative estimate for $P(X)$. Actually, the values of $\bar{p}_{X}^{0.95}$ and $d_{X}$ are $2.5923 \cdot 10^{-2}$ and 72.7040 , respectively, i.e., 35.87 and 44.14 times larger than those obtained under the 'baseline' assumption of objective independence between all the BEs (Configuration T1).

\begin{tabular}{|c|c|c|c|}
\hline \multicolumn{4}{|c|}{$\begin{array}{l}\text { Analysis } 1 \text { - Unknown epistemic dependence between the probabilities (chances) of the BEs } \\
\text { Case B }\end{array}$} \\
\hline \multirow[b]{2}{*}{ Top Event (TE) $X$ (configuration, Table III) } & \multicolumn{3}{|c|}{ Indicators } \\
\hline & $E\left[\boldsymbol{P}(\boldsymbol{X})^{I N S}\right]$ & $d_{X}$ & $p_{X}^{0.95}, \bar{p}_{X}^{0.95}$ \\
\hline$X=\left[\left(B_{1} \cup_{\text {ind }} B_{2} \cup_{\text {ind }} B_{3}\right) \cap_{\text {ind }}\left(B_{4} \cup_{\text {ind }} B_{5} \cup_{\text {ind }} B_{6}\right)\right]^{u k n}(\mathrm{~T} 1)$ & $2.8725 \cdot 10^{-4}$ & 1.6472 & {$\left[7.3617 \cdot 10^{-5}, 7.2275 \cdot 10^{-4}\right]$} \\
\hline Positive (pos) objective dependence between $B_{1}$ and $B_{6}(\mathrm{~T} 2)$ & $2.2574 \cdot 10^{-3}$ & 15.3945 & {$\left[6.5629 \cdot 10^{-5}, 8.9766 \cdot 10^{-3}\right]$} \\
\hline$X=\left[\left(B_{1} \cup_{\text {ind }} B_{2} \cup_{\text {ind }} B_{3}\right) \cap_{\text {ind }}\left(B_{4} \cup_{u k n} B_{5} \cup_{\text {ind }} B_{6}\right)\right]^{u k n}(\mathrm{~T} 3)$ & $2.8998 \cdot 10^{-4}$ & 1.7324 & {$\left[6.1237 \cdot 10^{-5}, 7.7580 \cdot 10^{-4}\right]$} \\
\hline$X=\left[\left(B_{1} \cup_{u k n} B_{2} \cup_{u k n} B_{3}\right) \cap_{u k n}\left(B_{4} \cup_{u k n} B_{5} \cup_{u k n} B_{6}\right)\right]^{u k n}(\mathrm{~T} 4)$ & $1.0463 \cdot 10^{-2}$ & 72.7040 & {$\left[3.5735 \cdot 10^{-5}, 2.5923 \cdot 10^{-2}\right]$} \\
\hline
\end{tabular}


Table V. Values of the indicators $\left[\underline{p}_{X}^{0.95}, \bar{p}_{X}^{0.95}\right]$ (19) and $d_{X}(20)$ obtained for $P(X)$ under different assumptions of objective dependence between the BEs (Configurations T1-T4 in Table III), with reference to Case B; the estimates for $E\left[P(X)^{I N S}\right]$ are also reported

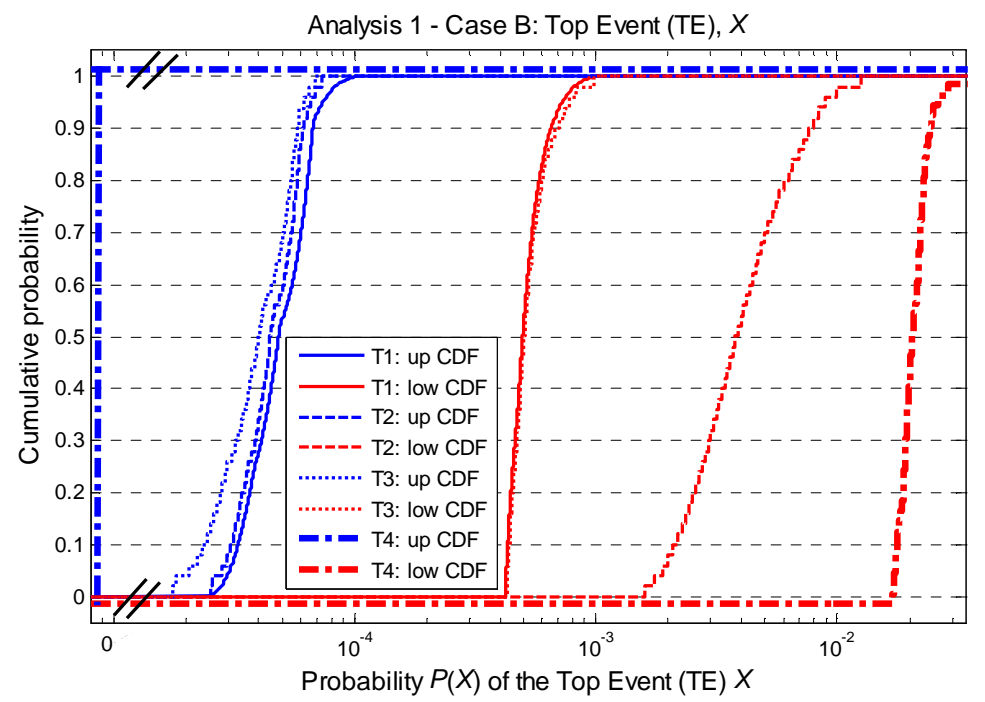

Figure 7. Upper and lower CDFs $\bar{F}^{P(X)}$ and $\underline{F}^{P(X)}$ obtained for $P(X)$, with reference to Case $B$ under different assumptions of objective dependence between the BEs (Configurations T1T4 in Table III). The value $P(X)=0$ is represented out of scale at about $1 \cdot 10^{-5}$ for clarity of illustration

Some considerations are in order with respect to the results obtained. It has been shown that the assumption of objective independence between BEs linked by AND-gates very often leads to a significant underestimation of i) the risk associated to the system (here represented by the upper bound $\bar{p}_{X}^{0.95}$ of the 95-th quantile $P(X)^{0.95}$ of $\left.P(X)\right)$ and ii) the uncertainty (imprecision) 'contained' in the distribution of $P(X)$ (here represented by the relative average distance $d_{X}$ between the upper and lower CDFs $\bar{F}^{P(X)}$ and $\underline{F}^{P(X)}$ of the TE probability-chance $\left.P(X)\right)$. In more detail, it can be seen that when the BE probabilities (chances) are of the order of $10^{-1}$ (like in the present Case A), the assumption of objective independence leads to underestimating risk and uncertainty by $2.22-2.27$ times and 2.61-4.21 times, respectively, with respect to the assumption of unknown objective dependence. Instead, if the $\mathrm{BE}$ probabilities (chances) are of the order of $10^{-2}-10^{-3}$ (like in the present Case B), the assumption of objective independence leads to underestimating risk and uncertainty by 89 
125 times and 136-164 times, respectively, with respect to the assumption of unknown objective dependence. Thus, the effects of objective dependences between BEs linked by AND-gates becomes more and more dramatic as the BE probabilities (chances) decrease: this poses serious concerns in the risk assessment of complex systems where the components are highly reliable and, thus, characterized by very small failure probabilities (chances).

Instead, it has been shown that the assumption of objective independence between BEs linked by OR-gates leads to a slight underestimation of both risk and uncertainty. In particular, it can be seen that when the BE probabilities (chances) are of the order of $10^{-1}$ (like in the present Case A), the assumption of objective independence leads to underestimating risk and uncertainty by $1.26-1.28$ times and $1.45-1.85$ times, respectively, with respect to the assumption of unknown objective dependence. Instead, if the BE probabilities (chances) are of the order of $10^{-2}-10^{-3}$ (like in the present Case B), the assumption of objective independence does not lead to a remarkable underestimation of risk, whereas it causes a non negligible underestimation of uncertainty (i.e., by 1.14-1.27 times with respect to the assumption of unknown objective dependence). Based on these considerations, it can be concluded that i) the assumption of objective independence between BEs linked by OR-gates leads to a slight underestimation of risk only when the BE probabilities (chances) are relatively large (e.g., of the order of $10^{-1}$ ) and ii) the relevance of the underestimation of uncertainty does not change dramatically as the BE probabilities (chances) change. These considerations makes the treatment of dependences between BEs linked by OR-gates much less critical than for AND-gates. 


\subsection{Studying the effects of state-of-knowledge (epistemic) dependences between the probabilities (chances) of the basic events}

Table VI reports the values of the indicators $\left[\underline{p}_{Z}^{0.95}, \bar{p}_{Z}^{0.95}\right]$ (19) and $d_{Z}(20)$ obtained for the events $Z=\left(B_{1} \cap_{\text {ind }} B_{6}\right)^{\text {epi }},\left(B_{1} \bigcap_{\text {ind }} B_{5}\right)^{e p i},\left(B_{2} \bigcap_{\text {ind }} B_{5}\right)^{e p i},\left(B_{4} \cup_{\text {ind }} B_{5}\right)^{e p i}$ and $\left(B_{2} \cup_{\text {ind }} B_{3}\right)^{e p i}$ (Configurations C1-C5 of Analysis 2 in Table III) under the assumptions of independence ('epi' = 'ind'), perfect ('epi' = 'perf') and unknown ('epi' = 'ukn') epistemic dependence, with reference to Cases $\mathrm{A}$ and $\mathrm{B}$; the estimates for $E\left[P(Z)^{I N S}\right]$ are also reported for completeness. In addition, only for illustration purposes, Figure 8 shows the upper and lower Cumulative Distribution Functions (CDFs) $\bar{F}^{P\left[\left(B_{1} \cap_{\text {ind }} B_{5}\right)^{e p i}\right]}, \bar{F}^{P\left[\left(B_{4} \cup_{\text {ind }} B_{5}\right)^{e p i}\right]}, \underline{F}^{P\left[\left(B_{1} \cap_{\text {ind }} B_{5}\right)^{e p i}\right]}$ and $\underline{F}^{P\left[\left(B_{4} \cup_{\text {ind }} B_{5}\right)^{e p i}\right]}$ obtained for events $\left(B_{1} \cap_{\text {ind }} B_{5}\right)^{e p i}$ (top) and $\left(B_{4} \cup_{\text {ind }} B_{5}\right)^{e p i}$ (bottom), respectively, under the assumptions of independence (solid lines), perfect (dashed lines) and unknown (dot-dashed lines) epistemic dependence, with reference to Cases A (left) and B (right).

We start by analyzing the cases where the BEs are linked by AND-gates and we refer only to event $\mathrm{Z}=\left(B_{1} \cap_{\text {ind }} B_{5}\right)^{\text {epi }}(\mathrm{C} 2)$ for brevity sake. It can be seen that in Case A the values of the upper bound $\bar{p}_{\left(B_{1} \cap_{\text {ind }} B_{5}\right)^{e p i}}^{0.95}$ of the 95-th percentile $P\left[\left(B_{1} \cap_{\text {ind }} B_{5}\right)^{e p i}\right]^{0.95}$ are $0.1216,0.1467$ and 0.1557 under the assumptions of independence, total (perfect) and unknown epistemic dependence, respectively. Thus, the assumption of epistemic independence would lead to underestimating the upper bound of the 95-th quantile (and, thus, the risk associated to the system) by 1.21 and 1.28 times with respect to the assumptions of total and unknown epistemic dependence, respectively; in addition, notice that the assumption of perfect dependence produces estimates of the upper bound of the 95-th quantile that are comparable to those obtained under the assumption of unknown dependence. These considerations are 
reflected also by the analysis of the values of the relative average distance $d_{\left(B_{1} \cap_{\text {ind }} B_{5}\right)^{e p i}}$ between the upper and lower CDFs $\bar{F}^{P\left[\left(B_{1} \cap_{\text {ind }} B_{5}\right)^{e p i}\right]}$ and $\underline{F}^{P\left[\left(B_{1} \cap_{\text {ind }} B_{5}\right)^{e p i}\right]}$. Actually, as before the assumption of epistemic independence leads to underestimating the uncertainty (imprecision) 'contained' in the distribution of $P\left[\left(B_{1} \cap_{\text {ind }} B_{5}\right)^{e p i}\right]$ by about 1.04 and 1.92 times with respect to the assumptions of perfect and unknown epistemic dependence, respectively. Similar considerations can be drawn from the analyses of events $\left(B_{1} \cap_{\text {ind }} B_{6}\right)^{e p i}$ and $\left(B_{2} \cap_{\text {ind }} B_{5}\right)^{e p i}$. No significant differences can be found here between the results obtained in Cases A and B. For example, in Case B, the assumption of epistemic independence leads to underestimating the upper bounds of the 95-th quantiles (and, thus, the risk associated to the system) by 1.287 and 1.290 times with respect to the assumptions of total and unknown epistemic dependence, respectively; in addition, the estimates produced by the assumptions of total and unknown epistemic dependence are almost identical as before.

Very similar considerations (and results) can be drawn by the analysis of those cases where the BEs are linked by OR-gates, i.e., $Z=\left(B_{4} \cup{ }_{\text {ind }} B_{5}\right)^{e p i}$ and $\left(B_{2} \cup_{\text {ind }} B_{3}\right)^{e p i}$ (Configurations C4 and C5 in Table III) in both Cases A and B: thus, we analyze only event $\left(B_{4} \cup \text { ind } B_{5}\right)^{e p i}$ with reference to Case A for brevity. It can be seen that the assumption of independence leads to underestimating the upper bounds of the 95-th quantiles (and, thus, the risk associated to the system) by 1.03 and 1.04 times with respect to the assumptions of total and unknown dependence, respectively.

These results are pictorially confirmed by Figure 8: actually, it can be seen that the upper and lower CDFs of $P\left[\left(B_{1} \cap_{\text {ind }} B_{5}\right)^{e p i}\right]$ (top) and $P\left[\left(B_{4} \cup_{\text {ind }} B_{5}\right)^{e p i}\right]$ (bottom) obtained under the assumption of unknown epistemic dependence (dot-dashed lines) completely envelop those obtained under the assumptions of independence (solid lines) and perfect dependence (dashed lines) in both Cases A (left) and B (right) (i.e., they obviously represent more conservative estimates of the bounding distributions). In addition, it is worth noting that the lower (resp., 
upper) CDFs obtained under the assumption of perfect epistemic dependence, i.e., $\underline{F}^{P\left[\left(B_{1} \cap_{\text {ind }} B_{5}\right)^{\text {perf }}\right]}$ and $\underline{F}^{P\left[\left(B_{4} \cup \text { ind } B_{5}\right)^{\text {perf }}\right]}$ (resp., $\bar{F}^{P\left[\left(B_{1} \cap_{\text {ind }} B_{5}\right)^{\text {perf }}\right]}$ and $\bar{F}^{P\left[\left(B_{4} \cup \text { ind } B_{5}\right)^{\text {perf }}\right]}$ ), are very close to those produced by the assumption of unknown epistemic dependence, i.e., $\underline{F}^{P\left[\left(B_{1} \cap_{\text {ind }} B_{5}\right)^{u k n}\right]}$ and $\underline{F}^{P\left[\left(B_{4} \cup_{\text {ind }} B_{5}\right)^{\mu k n}\right]}$ (resp., $\bar{F}^{P\left[\left(B_{1} \cap_{\text {ind }} B_{5}\right)^{\mu k n}\right]}$ and $\bar{F}^{P\left[\left(B_{4} \cup_{\text {ind }} B_{5}\right)^{\mu k n}\right]}$ ) in the region where the cumulative probability is very close to the 'extreme' upper bound 1 (resp., lower bound 0). In other words, the CDFs produced under assumptions of perfect and unknown epistemic dependence are almost identical in the range of extreme probabilities-chances (i.e., extreme quantiles) that are of particular interest in the risk assessment of complex, highly reliable systems.

\begin{tabular}{|c|c|c|c|c|}
\hline \multicolumn{5}{|c|}{ Analysis 2 - Objective independence (ind) between the BEs } \\
\hline \multicolumn{5}{|c|}{ Case A } \\
\hline & & \multicolumn{3}{|c|}{ State of epistemic (epi) dependence } \\
\hline Event $Z$ & Indicators & Independence (ind) & Perfect (perf) & Unknown $(u k n)$ \\
\hline \multirow{3}{*}{$\begin{array}{c}\left(\boldsymbol{B}_{1} \cap_{\text {ind }} \boldsymbol{B}_{6}\right)^{e p i} \\
(\mathbf{C} \mathbf{1})\end{array}$} & $E\left[P(Z)^{I N S}\right]$ & 0.0543 & 0.0576 & 0.0583 \\
\hline & $d_{z}$ & 0 & 0 & 0.9270 \\
\hline & {$\left[\underline{p}_{Z}^{0.95}, \overline{\boldsymbol{p}}_{Z}^{0.95}\right.$} & 0.0974 & 0.1230 & {$[0.0492,0.1528]$} \\
\hline \multirow{3}{*}{$\begin{array}{c}\left(\boldsymbol{B}_{1} \cap_{\text {ind }} \boldsymbol{B}_{5}\right)^{e p i} \\
(\mathbf{C} 2)\end{array}$} & $\boldsymbol{E}\left[\boldsymbol{P}(\boldsymbol{Z})^{I N S}\right]$ & 0.0474 & 0.0510 & 0.0553 \\
\hline & $d_{z}$ & 0.8993 & 0.9389 & 1.7242 \\
\hline & $\underline{p}_{Z}^{0.95}, \bar{p}_{Z}^{0.95}$ & {$[0.0503,0.1216]$} & {$[0.0634,0.1467]$} & {$[0.0243,0.1557]$} \\
\hline \multirow{3}{*}{$\begin{array}{c}\left(\boldsymbol{B}_{2} \bigcap_{\text {ind }} \boldsymbol{B}_{5}\right)^{e p i} \\
(\mathbf{C} 3)\end{array}$} & $\boldsymbol{E}\left[\boldsymbol{P}(\boldsymbol{Z})^{I N S}\right]$ & 0.0609 & 0.0622 & 0.0715 \\
\hline & $d_{z}$ & 1.4874 & 1.5152 & 1.9523 \\
\hline & {$\left[\underline{p}_{Z}^{0.95}, \overline{\boldsymbol{p}}_{Z}^{0.95}\right.$} & {$[0.0254,0.1551]$} & {$[0.0283,0.1717]$} & {$[0.0188,0.1760]$} \\
\hline \multirow{3}{*}{$\begin{array}{c}\left(\boldsymbol{B}_{4} \cup_{\text {ind }} \boldsymbol{B}_{5}\right)^{e p i} \\
(\mathbf{C} 4)\end{array}$} & $E\left[\boldsymbol{P}(\boldsymbol{Z})^{I N S}\right]$ & 0.3817 & 0.3740 & 0.3933 \\
\hline & $d_{z}$ & 0.6510 & 0.6393 & 0.9608 \\
\hline & $\underline{p}_{z}^{0.95}, \bar{p}_{z}^{0.95}$ & {$[0.3786,0.6423]$} & {$[0.3933,0.6618]$} & {$[0.3100,0.6670]$} \\
\hline \multirow{3}{*}{$\begin{array}{c}\left(\boldsymbol{B}_{2} \cup_{\text {ind }} \boldsymbol{B}_{3}\right)^{e p i} \\
(\mathbf{C} 5)\end{array}$} & $E\left[P(Z)^{I N S}\right]$ & 0.5192 & 0.5178 & 0.5272 \\
\hline & $d_{z}$ & 0.4239 & 0.4229 & 0.5241 \\
\hline & $\underline{p}_{Z}^{0.95}, \overline{\boldsymbol{p}}_{Z}^{0.95}$ & {$[0.4707,0.6798]$} & {$[0.4813,0.6932]$} & {$[0.4519,0.6970]$} \\
\hline \multicolumn{5}{|c|}{ Case B } \\
\hline & & \multicolumn{3}{|c|}{ State of epistemic (epi) dependence } \\
\hline Event $Z$ & Indicators & Independence (ind) & Perfect (perf) & Unknown $(u k n)$ \\
\hline \multirow{3}{*}{$\begin{array}{c}\left(\boldsymbol{B}_{1} \cap_{\text {ind }} \boldsymbol{B}_{6}\right)^{e p i} \\
(\mathbf{C 1})\end{array}$} & $\boldsymbol{E}\left[\boldsymbol{P}(\boldsymbol{Z})^{I N S}\right]$ & $1.62 \cdot 10^{-5}$ & $2.01 \cdot 10^{-5}$ & $2.01 \cdot 10^{-5}$ \\
\hline & $d_{z}$ & 0 & 0 & 1.6603 \\
\hline & {$\left[\underline{\boldsymbol{p}}_{Z}^{0.95}, \overline{\boldsymbol{p}}_{Z}^{0.95}\right.$} & $4.36 \cdot 10^{-5}$ & $6.72 \cdot 10^{-5}$ & {$\left[1.41 \cdot 10^{-5}, 9.21 \cdot 10^{-5}\right]$} \\
\hline \multirow{2}{*}{$\begin{array}{c}\left(\boldsymbol{B}_{1} \cap_{\text {ind }} \boldsymbol{B}_{5}\right)^{e p i} \\
(\mathbf{C} 2)\end{array}$} & $\boldsymbol{E}\left[\boldsymbol{P}(\boldsymbol{Z})^{I N S}\right]$ & $1.69 \cdot 10^{-5}$ & $1.96 \cdot 10^{-5}$ & $1.85 \cdot 10^{-5}$ \\
\hline & $d_{z}$ & 0.9112 & 0.9943 & 1.3109 \\
\hline
\end{tabular}




\begin{tabular}{|c|c|c|c|c|}
\hline & {$\left[\underline{p}_{z}^{0.95}, \bar{p}_{z}^{0.95}\right]$} & {$\left[2.38 \cdot 10^{-5}, 6.09 \cdot 10^{-5}\right]$} & {$\left[3.45 \cdot 10^{-5}, 7.85 \cdot 10^{-5}\right]$} & {$\left[8.75 \cdot 10^{-6}, 7.88 \cdot 10^{-5}\right]$} \\
\hline \multirow{3}{*}{$\begin{array}{c}\left(\boldsymbol{B}_{2} \cap_{\text {ind }} \boldsymbol{B}_{5}\right)^{e p i} \\
(\mathbf{C} \mathbf{3})\end{array}$} & $\boldsymbol{E}\left[\boldsymbol{P}(\boldsymbol{Z})^{I N S}\right]$ & $2.43 \cdot 10^{-5}$ & $2.49 \cdot 10^{-5}$ & $2.62 \cdot 10^{-5}$ \\
\hline & $d_{z}$ & 1.4878 & 1.5251 & 1.8371 \\
\hline & $\underline{\boldsymbol{p}}_{Z}^{0.95}, \overline{\boldsymbol{p}}_{\mathrm{Z}}^{0.95}$ & {$\left[1.01 \cdot 10^{-5}, 6.19 \cdot 10^{-5}\right]$} & {$\left[1.13 \cdot 10^{-5}, 6.87 \cdot 10^{-5}\right]$} & {$\left[5.68 \cdot 10^{-6}, 7.01 \cdot 10^{-5}\right]$} \\
\hline \multirow{3}{*}{$\begin{array}{c}\left(\boldsymbol{B}_{4} \cup_{\text {ind }} \boldsymbol{B}_{5}\right)^{e p i} \\
(\mathbf{C 4})\end{array}$} & $\boldsymbol{E}\left[\boldsymbol{P}(\boldsymbol{Z})^{I N S}\right]$ & $8.69 \cdot 10^{-3}$ & $8.65 \cdot 10^{-3}$ & $8.93 \cdot 10^{-3}$ \\
\hline & $d_{z}$ & 0.7266 & 0.7237 & 1.0499 \\
\hline & $\underline{\boldsymbol{p}}_{z}^{0.95}, \overline{\boldsymbol{p}}_{z}^{0.95}$ & {$\left[8.38 \cdot 10^{-3}, 1.59 \cdot 10^{-2}\right]$} & {$\left[8.82 \cdot 10^{-3}, 1.67 \cdot 10^{-2}\right]$} & {$\left[6.59 \cdot 10^{-3}, 1.67 \cdot 10^{-2}\right]$} \\
\hline \multirow{3}{*}{$\begin{array}{c}\left(\boldsymbol{B}_{2} \cup_{\text {ind }} \boldsymbol{B}_{3}\right)^{e p i} \\
(\mathbf{C} 5)\end{array}$} & $\boldsymbol{E}\left[\boldsymbol{P}(Z)^{I N S}\right]$ & $1.22 \cdot 10^{-2}$ & $1.22 \cdot 10^{-2}$ & $1.25 \cdot 10^{-2}$ \\
\hline & $d_{z}$ & 0.5289 & 0.5298 & 0.6502 \\
\hline & $p_{Z}^{0.95}, \bar{p}_{Z}^{0.95}$ & {$\left[1.05 \cdot 10^{-2}, 1.72 \cdot 10^{-2}\right]$} & {$\left[1.07 \cdot 10^{-2}, 1.76 \cdot 10^{-2}\right]$} & {$\left[9.83 \cdot 10^{-3}, 1.78 \cdot 10^{-2}\right]$} \\
\hline
\end{tabular}

Table VI. Values of the indicators $\left[\underline{p}_{Z}^{0.95}, \bar{p}_{Z}^{0.95}\right]$ (19) and $d_{Z}(20)$ obtained for events $Z=\left(B_{1}\right.$ $\left.\bigcap_{\text {ind }} B_{6}\right)^{e p i},\left(B_{1} \cap_{\text {ind }} B_{5}\right)^{e p i},\left(B_{2} \bigcap_{\text {ind }} B_{5}\right)^{e p i},\left(B_{4} \cup \cup_{\text {ind }} B_{5}\right)^{e p i}$ and $\left(B_{2} \cup \text { ind } B_{3}\right)^{e p i}$ (Configurations

C1-C5 of Analysis 2 in Table III) under the assumptions of independence, perfect and unknown epistemic dependence, with reference to Cases $A$ and $B$

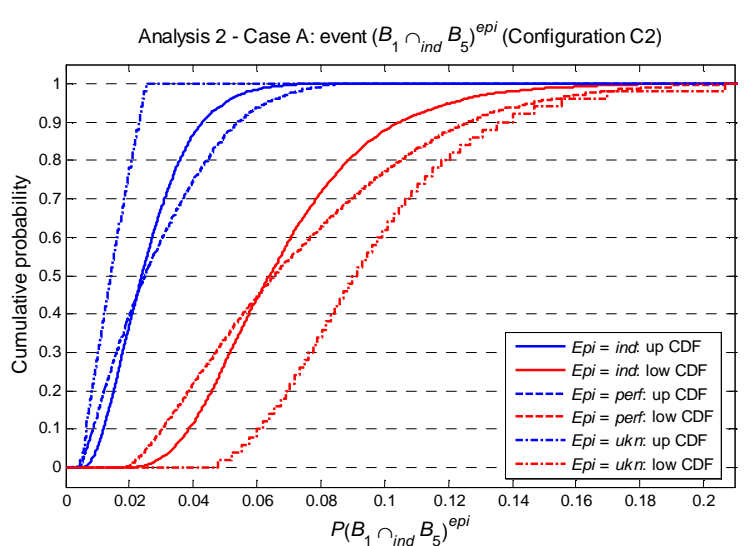

Analysis 2 - Case A: event $\left(B_{4} \cup \text { ind } B_{5}\right)^{e p i}($ Configuration C4)

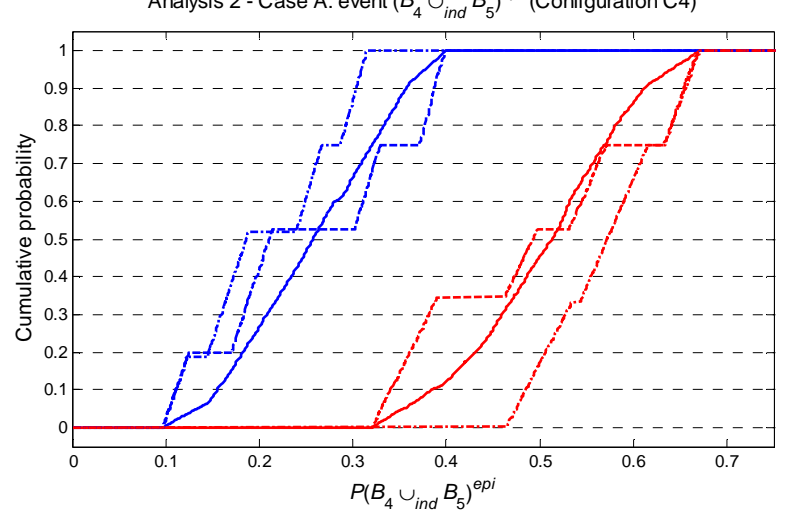

Figure 8. Upper and lower CDFs $\bar{F}^{P\left[\left(B_{1} \cap_{\text {ind }} B_{5}\right)^{e p i}\right]}, \bar{F}^{P\left[\left(B_{4} \cup_{\text {ind }} B_{5}\right)^{e p i}\right]}, \underline{F}^{P\left[\left(B_{1} \cap_{\text {ind }} B_{5}\right)^{e p i}\right]}$ and $\underline{F}^{P\left[\left(B_{4} \cup_{\text {ind }} B_{5}\right)^{e p i}\right]}$ obtained for events $\left(B_{1} \cap_{\text {ind }} B_{5}\right)^{\text {epi }}$ (top) and $\left(B_{4} \cup_{\text {ind }} B_{5}\right)^{\text {epi }}$ (bottom), respectively, under the assumptions of independence (solid lines), perfect (dashed lines) and unknown (dot-dashed lines) epistemic dependence, with reference to Cases A (left) and B (right)
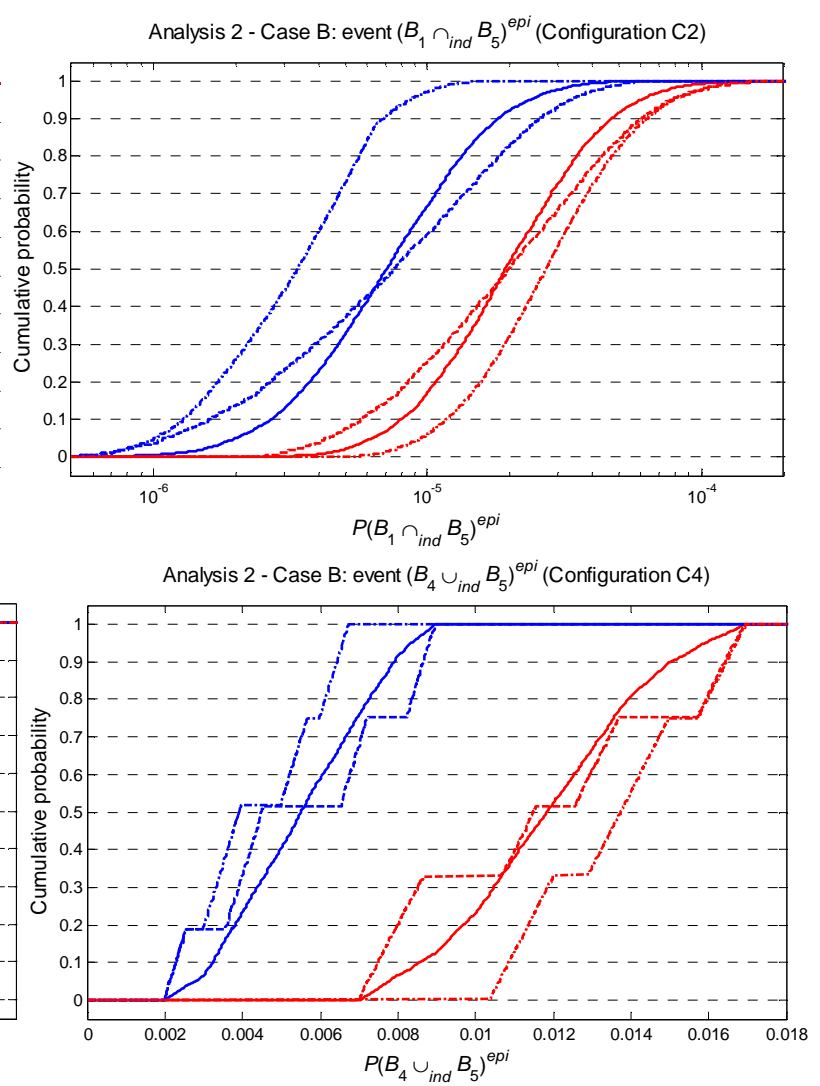
Similar analyses performed on $P(X)$. Table VII reports the values of the indicators $\left[\underline{p}_{X}^{0.95}, \bar{p}_{X}^{0.95}\right](19)$ and $d_{X}(20)$ obtained for $P(X)$ under different assumptions of epistemic dependence between the probabilities (chances) of the BEs (Configurations T1-T3 of Analysis 2 in Table III), with reference to Case B; the estimates for $E\left[P(X)^{I N S}\right]$ are also shown for completeness. For illustration purposes, Figure 9 depicts the upper and lower CDFs $\bar{F}^{P(X)}$ and $\underline{F}^{P(X)}$ obtained for $P(X)$ assuming independence (solid lines), perfect (dashed lines) and unknown (dot-dashed lines) epistemic dependence between the probabilities (chances) of all the BEs (Configurations T1-T3 of Analysis 2 in Table III).

It can be seen that the values of the upper bound $\bar{p}_{X}^{0.95}$ of the 95 -th percentile $P(X)^{0.95}$ are $4.4030 \cdot 10^{-4}, 6.4111 \cdot 10^{-4}$ and $7.2275 \cdot 10^{-4}$ under the assumptions of independence, total dependence and unknown dependence, respectively. Thus, the assumption of independence would lead to underestimating the upper bound of the 95-th quantile (and, thus, the risk associated to the system) by 1.456 and 1.641 times with respect to the assumptions of total and unknown dependence, respectively. This is reflected by the analysis of the indicator $d_{X}$ : the assumption of epistemic independence leads to underestimating $d_{X}$ by 1.02 and 2.56 times, with respect to the assumptions of total and unknown epistemic dependence.

\begin{tabular}{|c|c|c|c|}
\hline \multicolumn{4}{|c|}{$\begin{array}{l}\text { Analysis } 2 \text { - Objective independence (ind) between the BEs } \\
\text { Case B }\end{array}$} \\
\hline & \multicolumn{3}{|c|}{ Indicators } \\
\hline Top Event (TE) $X$ (configuration, Table III) & $E\left[\boldsymbol{P}(\boldsymbol{X})^{I N S}\right]$ & $d_{X}$ & $p_{X}^{0.95}, \bar{p}_{X}^{0.95}$ \\
\hline$X=\left[\left(B_{1} \cup{ }_{\text {ind }} B_{2} \cup_{\text {ind }} B_{3}\right) \cap_{\text {ind }}\left(B_{4} \cup_{\text {ind }} B_{5} \cup_{\text {ind }} B_{6}\right)\right]^{\text {ind }}(\mathrm{T} 1)$ & $2.1571 \cdot 10^{-4}$ & 0.8576 & {$\left[1.9821 \cdot 10^{-4}, 4.4030 \cdot 10^{-4}\right]$} \\
\hline$X=\left[\left(B_{1} \cup \cup_{\text {ind }} B_{2} \cup_{\text {ind }} B_{3}\right) \cap_{\text {ind }}\left(B_{4} \cup_{\text {ind }} B_{5} \cup_{\text {ind }} B_{6}\right)\right]^{\text {perf }}(\mathrm{T} 2)$ & $2.3119 \cdot 10^{-4}$ & 0.8781 & {$\left[3.1555 \cdot 10^{-4}, 6.4111 \cdot 10^{-4}\right]$} \\
\hline$X=\left[\left(B_{1} \cup \cup_{\text {ind }} B_{2} \cup_{\text {ind }} B_{3}\right) \cap_{\text {ind }}\left(B_{4} \cup_{\text {ind }} B_{5} \cup_{\text {ind }} B_{6}\right)\right]^{u k n}(\mathrm{~T} 3)$ & $2.8725 \cdot 10^{-4}$ & 2.1935 & {$\left[7.3617 \cdot 10^{-5}, 7.2275 \cdot 10^{-4}\right]$} \\
\hline
\end{tabular}

Table VII. Values of the indicators $\left[\underline{p}_{X}^{0.95}, \bar{p}_{X}^{0.95}\right]$ (19) and $d_{X}(20)$ obtained for $P(X)$ under the assumptions of independence, perfect and unknown epistemic dependence (Configurations T1-T3 of Analysis 2 in Table III), with reference to Cases A and B 


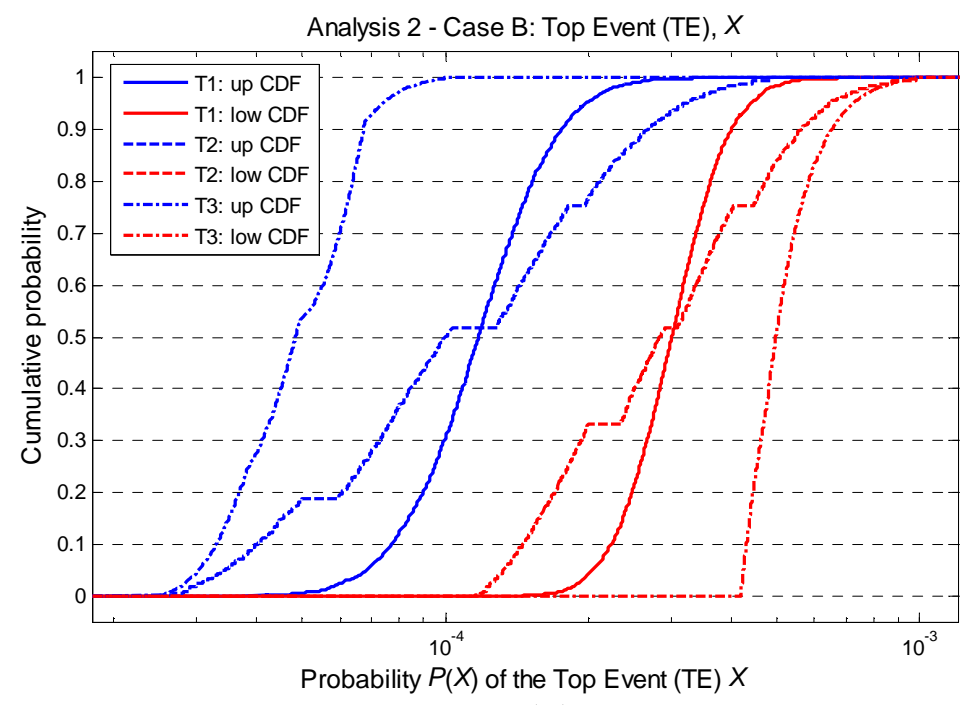

Figure 9. Upper and lower CDFs $\bar{F}^{P(X)}$ and $\underline{F}^{P(X)}$ obtained for $P(X)$ under the assumptions of independence (solid lines), perfect (dashed lines) and unknown (dot-dashed lines) epistemic dependence (Configurations T1-T3 of Analysis 2 in Table III), with reference to

Case $B$

Some considerations are in order with respect to the results obtained. It has been shown that the assumption of epistemic independence between the probabilities (chances) of BEs linked by AND-gates very often leads to an underestimation of i) the risk associated to the system (here represented by the upper bound of the 95-th quantile of the TE probability-chance) and ii) the 'imprecision' contained in the distribution of the TE probability-chance (here represented by the relative average distance between the upper and lower CDFs of the TE probability-chance). In particular, in the analysis of Configurations C1-C5 it is shown that when the BE probabilities (chances) are of the order of $10^{-1}$ (like in the present Case A), the assumption of epistemic independence leads to underestimating risk and uncertainty by $1.11-$ 1.57 times and 1.02-1.92 times, respectively, with respect to the assumptions of total and unknown epistemic dependence. Similarly, if the BE probabilities (chances) are of the order of $10^{-2}-10^{-3}$ (like in the present Case B), the assumption of epistemic independence leads to underestimating risk and uncertainty by 1.11-2.10 times and 1.03-1.44 times, respectively, with respect to the assumptions of total and unknown epistemic dependence. 
Similar results are obtained for BEs linked by OR-gates. In particular, it can be seen that when the BE probabilities (chances) are of the order of $10^{-1}$ (like in the present Case A), the assumption of independence leads to underestimating risk and uncertainty by $1.02-1.04$ times and 1.01-1.48 times, respectively, with respect to the assumptions of total and unknown epistemic dependence. If the BE probabilities (chances) are of the order of $10^{-2}-10^{-3}$ (like in the present Case B), the assumption of epistemic independence leads to underestimating risk and uncertainty by 1.025-1.05 times and 1.01-1.44 times, respectively, with respect to the assumptions of total and unknown epistemic dependence.

Finally, in the analysis of the probability (chance) of the TE of the FT in Figure 4 it is shown that assuming epistemic independence between the probabilities (chances) of all the BEs leads to underestimating risk and uncertainty by 1.456-1.641 and 1.02-2.56 times, respectively, with respect to the assumptions of total and unknown epistemic dependence. A final remark is in order with respect to the fact that in all the cases considered, the 95-th quantile estimates produced under the assumption of perfect dependence are comparable to those obtained under the hypothesis of unknown dependence.

On the basis of these considerations, it can be concluded that i) the effects of epistemic dependence are in general non negligible (in particular, in the estimation of small probabilities-chances and extreme quantiles), but they are quantitatively less relevant and critical than those of objective dependence (see the previous Section 4.1); ii) the effects of epistemic dependence are not influenced dramatically by the type of logical connection existing between the BEs, and iii) the effects of epistemic dependence are not modified significantly by the magnitude of the $\mathrm{BE}$ probabilities (chances). These considerations demonstrate that epistemic dependences cannot be neglected in the risk assessment of complex, safety-critical engineering systems (in particular, when small probabilities-chances 
and extreme quantiles have to be estimated); however, their effects are likely to be overwhelmed by those of objective dependences (if present).

\section{DISCUSSION AND CONCLUSIONS}

In this paper, the effects of objective and state-of-knowledge dependences between the BEs of a FT have been quantified. Two types of analyses have been carried out on a FT with six BEs:

1. assuming unknown epistemic dependence between the probabilities (chances) of the BEs, the effects of different states of objective dependence between the BEs have been quantified;

2. assuming objective independence between the BEs, the effects of different states of epistemic dependence between the probabilities (chances) of the BEs have been studied.

With respect to analysis 1 . above, it has been shown that:

- the assumption of objective independence between the BEs linked by AND-gates always leads to a serious underestimation of i) the risk associated to the system (here represented by the upper bound of the 95-th quantile of the TE probability-chance) and ii) the uncertainty (imprecision) 'contained' in the (distribution of the) TE probability-chance (here represented by the relative average distance between the upper and lower CDFs of the TE probability-chance) with respect to the assumptions of perfect and unknown objective dependence: actually, the corresponding estimates may differ even by several orders of magnitude;

- this underestimation becomes more and more dramatic as the BE probabilities (chances) get smaller: this poses serious concerns in the risk assessment of complex systems where the components are highly reliable and, thus, characterized by very small failure probabilities (chances); 
- the assumption of objective independence between BEs linked by OR-gates may lead to a slight underestimation of both risk and the uncertainty. In particular:

- the assumption of objective independence between BEs leads to a slight underestimation of risk only when the BE probabilities (chances) are relatively large (e.g., of the order of $10^{-1}$ ); otherwise, when the $\mathrm{BE}$ probabilities (chances) are quite small (e.g., of the order of $10^{-2}-10^{-3}$ ), the assumption of independence produces risk estimates that are comparable even to those provided by the assumption of unknown dependence;

- the assumption of objective independence between BEs always leads to a slight underestimation of the uncertainty (imprecision) 'contained' in the distribution of the TE probability (chance);

- the effects of objective dependence between BEs linked by OR-gates are not influenced dramatically by the magnitude of the BE probabilities (chances).

Based on the considerations above, it can be concluded that:

- the treatment of objective dependences between BEs linked by AND-gates is much more critical than for OR-gates;

- unknown (or, at least, perfect) objective dependence should be assumed between BEs linked by AND-gates, in particular if the corresponding probabilities (chances) are very small (e.g., of the order of $10^{-3}-10^{-2}$ ): this leads to obtaining conservative risk estimates;

- objective dependences between BEs linked by OR-gates can be in general neglected if the corresponding probabilities (chances) are very small (e.g., around $10^{-3}-10^{-2}$ ).

With respect to analysis 2 . above, it has been shown that: 
- the assumption of epistemic independence between the probabilities (chances) of the BEs leads to a non negligible underestimation of the risk associated to the system (here represented by the upper bound of the 95-th quantile of the TE probabilitychance) with respect to the assumptions of perfect and unknown epistemic dependence: this is particularly evident in the estimation of small probabilities (chances) and extreme quantiles that are of paramount importance in the risk assessment of complex, highly reliable systems;

- the estimates for the upper bound of the 95-th quantile of the TE probability (chance) produced by the assumptions of perfect and unknown epistemic dependence are comparable;

- the effects of epistemic dependence between the BE probabilities (chances) are quantitatively less relevant and critical than those of objective dependence between the BEs: they may differ by several orders of magnitude;

- the effects of epistemic dependence are not modified significantly by the magnitude of the BE probabilities (chances);

- the effects of epistemic dependence are not influenced dramatically by the type of logical connection existing between the BEs.

Based on the considerations above, it can be concluded that:

- the conditions of epistemic dependence between some BE probabilities (chances) should not be neglected when small probabilities (chances) and extreme quantiles have to be estimated: with respect to that, unknown (or, at least, perfect) epistemic dependences should be assumed in order to obtain conservative risk estimates;

- if objective dependences are also present (e.g., between BEs linked by AND-gates and characterized by very small probabilities-chances), the effects of epistemic dependence are likely to be overwhelmed by those of objective dependence. 


\section{ACKNOWLEDGMENTS}

The authors are grateful to the two anonymous reviewers for their useful comments and suggestions which have allowed improving the paper.

\section{REFERENCES}

1. Henley EJ, Kumamoto H. Probabilistic risk assessment. New York: IEEE Press, 1992.

2. USNRC. Guidance on the Treatment of Uncertainties Associated with PRAs in RiskInformed Decision Making. NUREG-1855, US Nuclear Regulatory Commission, Washington, DC, 2009.

3. NASA. Risk-Informed Decision Making Handbook. NASA/SP-2010-576 - Version 1.0, April 2010.

4. Epstein S, Rauzy A. Can we trust PRA? Reliability Engineering \& System Safety, 2005; 88(3): 195-205.

5. Cepin M. Analysis of truncation limit in probabilistic safety assessment. Reliability Engineering \& System Safety, 2005; 87(3): 395-403.

6. Lindley DV, Singpurwalla ND. Reliability (and fault tree) analysis using expert opinions. Journal of the American Statistical Association, 1986; 81(393): 87-90.

7. Apostolakis GE. The concept of probability in safety assessment of technological systems. Science, 1990; 250(4986): 1359-1364.

8. Aven T. Foundations of Risk Analysis: A Knowledge and Decision-Oriented Perspective. Chichester: Wiley, 2003.

9. Helton JC, Oberkampf W. Alternative representations of epistemic uncertainties. Reliability Engineering and System Safety, 2004; 85(1-3): 1-10. 
10. Limbourg P, de Rocquigny E. Uncertainty analysis using evidence theory - confronting level-1 and level-2 approaches with data availability and computational constraints, Reliability Engineering and System Safety, 2010; 95(5): 550-564.

11. Apostolakis GE, Kaplan S. Pitfalls in risk calculations. Reliability Engineering, 1981; 2(2): 135-145.

12. Huang D, Chen T, Wang MJ. A fuzzy set approach for event tree analysis. Fuzzy Sets and Systems, 2001; 118: 153-165.

13. NUREG-CR-6850. EPRI/NRC-RES Fire PRA methodology for nuclear power facilities, Volume 2: detailed methodology. US Nuclear Regulatory Commission, 2005.

14. USNRC. An approach for using probabilistic risk assessment in risk-informed decisions on plant-specific changes to the licensing basis. NUREG-1.174 - Revision 1, US Nuclear Regulatory Commission, Washington, DC, 2002.

15. Aven T. On the Need for Restricting the Probabilistic Analysis in Risk Assessments to Variability. Risk Analysis, 2010; 30(3): 354-360.

16. Aven T. Interpretations of alternative uncertainty representations in a reliability and risk analysis context. Reliability Engineering \& System Safety, 2011; 96(3): 353-360.

17. Aven T, Steen R. The concept of ignorance in a risk assessment and risk management context. Reliability Engineering and System Safety, 2010; 95(11): 1117-1122.

18. Aven T, Zio E. Some considerations on the treatment of uncertainties in risk assessment for practical decision making. Reliability Engineering and System Safety, 2010; 96(1): 64-74.

19. Ferson S, Ginzburg L, Akcakaya R. Whereof one cannot speak: when input distributions are unknown. Risk Analysis, 1996.

20. Klir GJ, Yuan B. Fuzzy Sets and Fuzzy Logic: Theory and Applications. Upper Saddle River, NJ: Prentice-Hall, 1995. 
21. Tanaka H, Fan LT, Lai FS. Fault tree analysis by fuzzy probability. IEEE Transactions on Reliability, 1983; 32: 453-457.

22. Liang GS, Wang MJJ. Fuzzy fault tree analysis using failure possibility. Microelectronics Reliability, 1993; 33(4): 583-597.

23. Huang HZ, Tong X, Zuo M. Posbist fault tree analysis of coherent systems. Reliability Engineering and System Safety, 2004; 84: 153-16.

24. Yuhua D, Datao Y. Estimation of failure probability of oil and gas transmission pipelines by fuzzy fault tree analysis. Journal of Loss Prevention in the Process Industries, 2005; 18: 83-88.

25. Ferdous R, Khan F, Veitch B, Amyotte P.R. Methodology for computer aided fuzzy fault tree analysis. Process safety and environmental protection, 2009; 87: 217-226.

26. Misra KB, Weber GG. A new method for fuzzy fault tree analysis. Microelectronics Reliability, 1989; 29(2): 195-216.

27. Soman KP, Misra KB. Fuzzy fault tree analysis using resolution identity and extension principle. International Journal of Fuzzy Mathematics, 1993; 1: 193-212.

28. Suresh PV, Babar AK, Venkat Raj V. Uncertainty in fault tree analysis: A fuzzy approach. Fuzzy Sets and Systems, 1996; 83: 135-141.

29. Baudrit C, Dubois D. Practical Representations of Incomplete Probabilistic Knowledge. Computational Statistics \& Data Analysis, 2006; 51(1): 86-108.

30. Baudrit C, Dubois D, Guyonnet D. Joint Propagation and Exploitation of Probabilistic and Possibilistic Information in Risk Assessment. IEEE Transactions on Fuzzy Systems, 2006; 14(5): 593-608.

31. Baudrit C, Dubois D, Perrot N. Representing parametric probabilistic models tainted with imprecision. Fuzzy Sets and System, 2008; 159(15): 1913-1928. 
32. Dubois D. Possibility theory and statistical reasoning. Computational Statistics \& Data Analysis, 2006; 51: 47-69.

33. Dubois D, Prade H. Possibility Theory: An Approach to Computerized Processing of Uncertainty. New York, NY: Plenum Press, 1988.

34. Baraldi P, Zio E. A Combined Monte Carlo and Possibilistic Approach to Uncertainty Propagation in Event Tree Analysis. Risk Analysis, 2008; 28(5): 1309-1326.

35. Flage R, Baraldi P, Ameruso F, Zio E, Aven T. Handling epistemic uncertainties in fault tree analysis by probabilistic and possibilistic approaches. Pp. 1761-1768 In: Bris R, Guedes Soares C, Martorell S. Reliability, Risk and Safety: Theory and Applications. Supplement Proceedings of the European Safety and Reliability Conference 2009 (ESREL 2009), Prague, Czech Republic, 7-10 September 2009, Boca Raton: Taylor \& Francis, 2010.

36. Flage R, Baraldi P, Zio E, Aven T. Possibility-probability transformation in comparing different approaches to the treatment of epistemic uncertainties in a fault tree analysis. Pp. 714-721 In: Ale B, Papazoglu IA, Zio E (Eds.). Reliability, Risk and Safety Proceedings of the European Safety and Reliability (ESREL) 2010 Conference, Rhodes, Greece, 5-9 September 2010. London, UK: Taylor \& Francis Group, 2010.

37. Limbourg P, Savic R, Petersen J, Kochs HD. Fault tree analysis in an early design stage using the Dempster-Shafer theory of evidence. Pp. 713-722 In: Aven and Vinnem (Eds.), Risk, Reliability and Societal Safety - Proceedings of the European Safety and Reliability (ESREL) 2007 Conference, Stavanger, Norway. London, UK: Taylor \& Francis Group, 2007.

38. Limbourg P, Savić R, Petersen J, Kochs H-D. Modelling uncertainty in fault tree analyses using evidence theory. Journal of Risk and Reliability, 2008; 222(3): 291-301. 
39. Ferson S, Kreinovich V, Ginzburg L, Sentz K, Myers DS. Constructing probability boxes and Dempster-Shafer structures. Sandia National Laboratories, Technical Report SAND2002-4015, Albuquerque, New Mexico, 2003.

40. Ferson S, Nelsen RB, Hajagos J, Berleant DJ, Zhang J, Tucker WT, Ginzburg LR, Oberkampf WL. Dependence in probabilistic modeling, Dempster-Shafer theory, and probability bounds analysis. Technical Report SAND2004-3072, Albuquerque, New Mexico, 2004.

41. Helton JC, Johnson JD, Oberkampf WL, Storlie CB. A sampling-based computational strategy for the representation of epistemic uncertainty in model predictions with evidence theory. Computer Methods in Applied Mechanics and Engineering, 2007; 196: 3980-3998.

42. Helton JC, Johnson JD, Oberkampf WL, Sallaberry CJ. Representation of Analysis Results Involving Aleatory and Epistemic Uncertainty. Sandia National Laboratories, Technical Report SAND2008-4379, Albuquerque, New Mexico, 2008.

43. Sentz K, Ferson S. Combination of Evidence in Dempster-Shafer Theory. Sandia National Laboratories, Technical Report SAND 2002-0835, Albuquerque, New Mexico, 2002.

44. Shafer G. A Mathematical Theory of Evidence. Princeton, NJ: Princeton University Press, 1976.

45. Ferson S, Hajagos JG. Arithmetic with uncertain numbers: rigorous and (often) best possible answers. Reliability Engineering and System Safety, 2004; 85: 135-152.

46. Ferson S, Tucker WT. Sensitivity in risk analyses with uncertain numbers. Technical Report SAND2006-2801, Setauket, New York 11733, 2006. 
47. Ferson S, Kreinovich V, Hajagos J, Oberkampf W, Ginzburg L. Experimental Uncertainty Estimation and Statistics for Data Having Interval Uncertainty. Technical Report SAND2007-0939, Setauket, New York 11733, 2007.

48. Ferson S, Van den Brink P, Estes TL, Gallagher K, O'Connor R, Verdonck F. Bounding uncertainty analyses. In: Warren-Hicks WJ, Hart A. Application of uncertainty analysis to ecological risks of pesticides. Pensacola and Boca Raton, FL: SETAC and CRC Press, 2010.

49. Moore RE. Methods and Applications of Interval Analysis. Philapdelphia, PA: SIAM, 1979.

50. USNRC. Procedure for analysis of common-cause failures in probabilistic safety analysis. Technical report NUREG/CR-5801 (SAND91-7087), 1993.

51. USNRC. Common-cause failure database and analysis system: event data collection, Classification, and coding. Technical report NUREG/CR-6268, 2007.

52. Zio E. Computational Methods for Reliability and Risk Analysis. World Scientific Publishing, 2009.

53. Watts DJ. Proc. Natl. Acad. Sci. U.S.A., 99, 5766, 2002.

54. Guimerà R, Arenas A, Dìaz-Guilera A, Giralt F. Dynamical Properties of Model Communication Networks. Phys. Rev. E, 2002; 66: 026704.

55. Sansavini G, Hajj MR, Puri IK, Zio E. A deterministic representation of cascade spreading in complex networks. EPL, 2009; 87 (4): art. no. 48004.

56. Zio E, Sansavini G. Modeling interdependent network systems for identifying cascadesafe operating margins. IEEE Transactions on Reliability, 2011; 60 (1): 94-101.

57. Zio E, Sansavini G. Component Criticality in Failure Cascade Processes of Network Systems. Risk Analysis, 2011; 31(8): 1196-1210. 
58. Fréchet M. Généralisations du théorème des probabilités totales. Fundamenta Mathematica, 1935; 25: 379-387.

59. Frank MJ, Nelsen RB, Schweizer B. Best-possible bounds for the distribution of a sum-a problem of Kolmogorov. Probability Theory and Related Fields, 1987; 74: 199-211.

60. Sadiq R, Saint-Martin E, Kleiner Y. Predicting risk of water quality failures in distribution networks under uncertainties using fault-tree analysis. Urban Water, 2008; 5(4): 287-304.

61. Berleant D, Goodman-Strauss C. Bounding the results of arithmetic operations on random variables of unknown dependency using intervals. Reliable Computing, 1998; 4 : 147-165.

62. Berleant D, Zhang J. Representation and problem solving with Distribution Envelope Determination (DEnv). Reliability Engineering and System Safety, 2004; 85: 153-168.

63. Berleant D, Zhang J. Using Pearson correlation to improve envelopes around the distributions of functions. Reliable Computing, 2004; 10: 139-161.

64. Berleant D, Xie L, Zhang J. Statool: a tool for distribution envelope determination (DEnv), an interval-based algorithm for arithmetic on random variables. Reliab Comput, 2003; 9(2): 91-108.

65. Berleant D, Anderson G, Goodman-Strauss C. Arithmetic on Bounded Families of Distributions: A DEnv Algorithm Tutorial. Pp. 183-210 In Hu C et al. (eds.). Knowledge Processing with Interval and Soft Computing. London, UK: Springer-Verlag, 2008.

66. Vaurio JK. Treatment of General Dependencies in System Fault-Tree and Risk Analysis. IEEE Transactions on Reliability, 2002; 51(3): 278-287.

67. Vaurio JK. Consistent mapping of common cause failure rates and alpha factors. Reliability Engineering and System Safety, 2007; 92(5): 628-645. 
68. Karanki DR, Dang VN. Quantification of uncertainty in fault tree analysis with correlated basic events. Pp. 1619-1628 In: Ale B, Papazoglu IA, Zio E (Eds.). Reliability, Risk and Safety - Proceedings of the European Safety and Reliability (ESREL) 2010 Conference, Rhodes, Greece, 5-9 September 2010. London, UK: Taylor \& Francis Group, 2010.

69. Li H. Hierarchical Risk Assessment of Water Supply Systems. Ph.D. thesis, Loughborough University, Leicestershire, UK, 2007.

70. Ferdous R, Khan F, Sadiq R, Amyotte P, Veitch B. Fault and Event Tree Analyses for Process Systems Risk Analysis: Uncertainty Handling Formulations. Risk Analysis, 2011; 31(1): 86-107.

71. Zhang Q. A general method dealing with correlations in uncertainty propagation in fault trees. Reliability Engineering and System Safety, 1989; 26(3): 231-247.

72. Zhang Q. A method dealing with correlations in uncertainty propagation by using traditional correlation coefficients. Reliability Engineering and System Safety, 1993; 41(2): 107-114.

73. Rushdi AM, Kafrawy KF. Uncertainty propagation in fault tree analyses using an exact method of moments. Microelectronics and Reliability, 1988; 28: 945-965.

74. Kafrawy KF, Rushdi AM. Uncertainty analysis of fault tree with statistically correlated failure data. Microelectronics and Reliability, 1990; 30: 157-175.

75. Karanki DR, Jadhav PA, Chandrakar A, Srividya A, Verma AK. Uncertainty analysis in PSA with correlated input parameters. Int J Syst Assur Eng Manag, 2010; 1: 66-71.

76. Huang B, Du X. A robust design method using variable transformation and GaussHermite integration. International Journal for Numerical Methods in Engineering, 2006; 66: $1841-1858$. 
77. Kalos MH, Whitlock PA. Monte Carlo methods. Volume I: Basics. New York, NY: Wiley, 1986.

78. Marseguerra M, Zio E. Basics of the Monte Carlo Method with Application to System Reliability. Hagen, Germany: LiLoLe-Verlag GmbH, 2002.

79. Karanki DR, Kushwaha HS, Verma AK, Ajit S. Uncertainty Analysis Based on Probability Bounds (P-Box) Approach in Probabilistic Safety Assessment. Risk Analysis, 2009; 29(5): 662-675.

80. Regan HM, Ferson S, Berleant D. Equivalence of five methods for bounding uncertainty. International Journal of Approximate Reasoning, 2004; 36: 1-30.

81. Tonon F. Using random set theory to propagate epistemic uncertainty through a mechanical system. Reliability Engineering and System Safety, 2004; 85: 169-181.

82. Couso I, Moral S. Independence concepts in evidence theory. International Journal of Approximate Reasoning, 2010; 51: 748-758.

83. Couso I, Moral S, Walley P. Examples of independence for imprecise probabilities. Pp. 121-130 In: de Cooman G, Cozman FF, Moral S, Walley P (eds.). Proceedings of the First International Symposium on Imprecise Probability and Their Applications. Imprecise Probabilities Project, Universiteit Gent, Belgium, 1999.

84. Couso I, Moral S, Walley P. A survey of concepts of independence for imprecise probabilities. Risk Decision and Policy, 2000; 5: 165-181.

85. Dubois D, Prade H, Sandri S. On possibility/probability transformations. Pp. 103-112 In: Lowen R, Roubens M (Eds.). Fuzzy Logic: State of the Art. Dordrecht, The Netherlands: Kluwer Academic Publishers, 1993.

86. Smets P. Constructing the pignistic probability function in a context of uncertainty. Pp. 29-39 In: Henrion M, Shachter RD, Kanal LN, Lemmer JF (eds). Uncertainty in Artificial Intelligence 5. North-Holland, 1990. 
87. Dubois D, Foulloy L, Mauris G, Prade H. Probability-possibility transformations, triangular fuzzy sets, and probabilistic inequalities. Reliable Computing, 2004; 10: 273 297.

88. Dubois D, Prade H, Smets P. A definition of subjective possibility. International Journal of Approximate Reasoning, 2008; 48: 352-364. 